\title{
Stokesian Dynamics simulation of Brownian suspensions
}

\author{
By THANH N. PHUNG ${ }^{1}$, JOHN F. BRADY \\ AND GEORGES BOSSIS ${ }^{2}$ \\ ${ }^{1}$ Division of Chemistry and Chemical Engineering, California Institute of Technology, \\ Pasadena, CA 91125, USA \\ ${ }^{2}$ Laboratoire de Physique de la Matière Condensée, Université de Nice Parc Valrose, \\ 06108 Nice Cedex, France
}

(Received 23 October 1995 and in revised form 21 December 1995)

The non-equilibrium behaviour of concentrated colloidal dispersions is studied by Stokesian Dynamics, a general molecular-dynamics-like technique for simulating particles suspended in a viscous fluid. The simulations are of a suspension of monodisperse Brownian hard spheres in simple shear flow as a function of the Péclet number, $P e$, which measures the relative importance of shear and Brownian forces. Three clearly defined regions of behaviour are revealed. There is first a Brownianmotion-dominated regime $(P e \leqslant 1)$ where departures from equilibrium in structure and diffusion are small, but the suspension viscosity shear thins dramatically. When the Brownian and hydrodynamic forces balance $(P e \approx 10)$, the dispersion forms a new 'phase' with the particles aligned in 'strings' along the flow direction and the strings are arranged hexagonally. This flow-induced ordering persists over a range of $P e$ and, while the structure and diffusivity now vary considerably, the rheology remains unchanged. Finally, there is a hydrodynamically dominated regime $(P e>200)$ with a dramatic change in the long-time self-diffusivity and the rheology. Here, as the Péclet number increases the suspension shear thickens owing to the formation of large clusters. The simulation results are shown to agree well with experiment.

\section{Introduction}

Suspensions of small particles dispersed in a fluid occur in a wide variety of natural and industrial settings, such as slurries, paints, pastes, dyes, polymers, many foodstuffs, and ceramic sols. In these microstructured fluids the suspended particles interact through hydrodynamic, interparticle, and Brownian (or thermal) forces. The balance between thermal and interparticle forces determines the equilibrium behaviour and gives rise to a variety of states from disordered amorphous gas or liquid microstructures with a relatively low shear viscosity to highly ordered crystalline dispersions with elastic moduli and yield stresses. Under the action of an external driving force such as shear, hydrodynamic forces come into play and compete with thermal and interparticle forces to set the structure and determine properties. An even richer variety of microstructures is now possible with flow-induced melting and ordering, and the transport properties in these highly non-equilibrium states can be vastly different from those at equilibrium. 
Our understanding of colloidal dispersions has increased markedly in the last decade. This has resulted in large part from three parallel developments: (i) excellent experiments on well-characterized model hard-sphere systems (de Kruif et al. 1985; van der Werff \& de Kruif 1989; van der Werff et al. 1989; Ackerson 1990, etc.), (ii) a recent scaling theory for the behaviour at high solids concentration (Brady 1993b), and 3) the invention of Stokesian Dynamics, a technique to numerically simulate suspension behaviour (Bossis \& Brady 1984, 1987, 1989; Brady \& Bossis 1985, 1988; Phung \& Brady 1992; Phung 1993). Stokesian Dynamics is a general molecular-dynamics-like method for simulating suspensions at low particle Reynolds number that accurately and efficiently computes the many-body interactions necessary to capture the hydrodynamic forces transmitted through the fluid.

In this work we report on simulation studies of rheology, diffusion, and structure of concentrated monodisperse suspensions of hard spheres. In a hard-sphere suspension particles interact through hydrodynamic and Brownian forces only, and the system is described by the minimal number of parameters: two, the volume fraction $\phi$ and the Péclet number, $P e$. The Péclet number is the ratio of hydrodynamic shear to thermal forces, or alternatively the ratio of Brownian to flow time scales; it is given by $P e=\dot{\gamma} a^{2} / D$, where $\dot{\gamma}$ is the magnitude of the shear rate, and $D=k T / 6 \pi \eta a$ is the diffusivity of an isolated spherical particle of radius $a$ and thermal energy $k T$ in a fluid of viscosity $\eta$. The studies presented here are restricted to a single volume fraction $\phi=0.45$, and thus the suspension behaviour depends on the single parameter $P e$.

As the Péclet number is varied the simulations reveal three characteristic regimes of suspension behaviour. At relatively low Péclet numbers $(P e \leqslant 1)$ the equilibrium structure is distorted by the flow and the suspension viscosity shear thins. The shear thinning is shown to be caused by the decrease of the direct Brownian contribution to the stress as the deformation of the structure cannot keep up with the flow. The hydrodynamic contribution to the stress remains constant and equal to the highfrequency dynamic viscosity throughout the shear thinning process. The simulation viscosities show no variation with the size or number of particles in a unit cell $(27 \leqslant N \leqslant 123)$ and compare well with experiment (van der Werff \& de Kruif 1989). Normal stress differences are also determined and are seen to be dominated by the direct Brownian contribution to the stress and increase as $P e$ increases. As is the case in many polymer systems, the first normal stress difference is positive and the second negative. Unlike polymer systems, however, both normal stress are of comparable magnitude.

At moderate Péclet numbers $(P e \approx 10)$ hydrodynamic and Brownian forces balance and the structure orders into 'strings' of particles aligned along the flow direction with the 'strings' arranged in an hexagonal array in the velocity-gradient-vorticity plane. This shear-induced ordering persists in the range $5<P e<200$, over which the suspension viscosity varies only slightly. The long-time self-diffusivity of a particle in the velocity-gradient direction drops dramatically by two orders of magnitude, however, as particle motion is confined to lie along the strings. This shear-induced ordering appears to be generic in any system with repulsive interparticle forces in simple shear flow (Bossis \& Brady 1984; Erpenbeck 1984; etc.). For Brownian particles, the variation in the relative mobility with separation of a pair of particles acts like a short-range repulsive force and is responsible for the ordering. This string ordering has been observed experimentally in this range of $P e$ and $\phi$ (Ackerson \& Pusey 1988; Ackerson 1990; Laun et al. 1992).

At higher $P e$ the ordered structure 'melts' as the shearing forces overcome Brownian motion and push particles into close contact where the short-range hydrodynamic 
lubrication forces are important. The melting appears to be gradual rather than sudden and no hysteresis was observed. After the structure has been destroyed the viscosity increases with increasing $P e$ - the suspension shear thickens. This shear thickening is due to the increase in the hydrodynamic contribution to the stress and is caused by the formation of large non-compact clusters that are bound by lubrication forces as first shown by Bossis \& Brady (1989). The first normal stress difference changes sign, and both normal stress differences appear to approach an $O(\eta \dot{\gamma})$ high- $P e$ asymptote. The long-time self-diffusivity also grows dramatically with $P e$ and reaches a purely hydrodynamic $O\left(\dot{\gamma} a^{2}\right)$ limit at high $P e$. The appearance of diffusive motion and normal stress differences in the deterministic pure hydrodynamic limit are surprising and is one of the reasons why we have delayed publishing these results. Recent theoretical work by Morris \& Brady (1996b) has shown, however, that the high-Pe limit is singular, and the residual effect of weak Brownian motion introduces irreversibility, which may result in finite normal stress differences and diffusive motion.

In the next section we outline the Stokesian Dynamics simulation method. In $\S 3$ we present and discuss the simulation results for rheology, diffusion, and structure in a concentrated colloidal dispersion of Brownian hard spheres at a volume fraction of 0.45 . Conclusions are given in $\S 4$.

\section{Simulation method}

A detailed derivation of the simulation method can be found elsewhere (Durlofsky, Brady \& Bossis 1987; Bossis \& Brady 1987, 1989; Brady \& Bossis 1988; Brady et al. 1988); here, we shall proceed quickly. For $N$ rigid particles suspended in an incompressible Newtonian fluid of viscosity $\eta$ and density $\rho$, the motion of the fluid is governed by the Navier-Stokes equations, while the particle motion is described by the coupled $N$-body Langevin equation:

$$
\boldsymbol{m} \cdot \frac{\mathrm{d} \boldsymbol{U}}{\mathrm{d} t}=\boldsymbol{F}^{H}+\boldsymbol{F}^{P}+\boldsymbol{F}^{B},
$$

In (2.1) $\boldsymbol{m}$ is the generalized mass/moment of inertia tensor, $U$ is the particle translational/rotational velocity vector of dimension $6 \mathrm{~N}$, and the $6 \mathrm{~N}$ force/torque vectors $\boldsymbol{F}$ represent: (i) the hydrodynamic forces $\boldsymbol{F}^{H}$ exerted on the particles due to their motion relative to the fluid, (ii) the deterministic non-hydrodynamic forces $\boldsymbol{F}^{P}$, which may be either interparticle or external, and (iii) the stochastic forces $\boldsymbol{F}^{\boldsymbol{B}}$ that give rise to Brownian motion.

When the motion is such that the particle Reynolds number is small, i.e. $R e=$ $\rho a^{2} \dot{\gamma} / \eta \ll 1$ for the shear flows considered here, the hydrodynamic force exerted on the particles in a suspension undergoing a bulk linear flow is

$$
\boldsymbol{F}^{H}=-\boldsymbol{R}_{F U} \cdot(\boldsymbol{U}-\langle\boldsymbol{U}\rangle)+\boldsymbol{R}_{F E}:\langle\boldsymbol{E}\rangle .
$$

In (2.2), $\langle\boldsymbol{U}\rangle=\langle\boldsymbol{\Gamma}\rangle \cdot \boldsymbol{x}$ is the imposed bulk flow evaluated at the particle centers, $\langle\boldsymbol{\Gamma}\rangle=\langle\boldsymbol{E}\rangle+\langle\boldsymbol{\Omega}\rangle$, and $\langle\boldsymbol{E}\rangle$ and $\langle\boldsymbol{\Omega}\rangle$ are the bulk rate-of-strain and vorticity tensors, respectively, and are constant in space but may be arbitrary functions of time. The configuration-dependent resistance tensors $\boldsymbol{R}_{F U}(\boldsymbol{x})$ and $\boldsymbol{R}_{F E}(\boldsymbol{x})$ give the hydrodynamic force/torque on the particles due to their motion relative to the fluid and due to an imposed flow, respectively. The vector $\boldsymbol{x}$ represents the generalized configuration vector specifying the location and orientation of all $N$ particles. 
The deterministic, non-hydrodynamic force $F^{P}$ is arbitrary and may be any form of interparticle or external force. The stochastic or Brownian force $F^{B}$ arises from the thermal fluctuations in the fluid and is characterized by

$$
\left\langle\boldsymbol{F}^{B}\right\rangle=0 \quad \text { and } \quad\left\langle\boldsymbol{F}^{B}(0) \boldsymbol{F}^{B}(t)\right\rangle=2 k T \boldsymbol{R}_{F U} \delta(t) .
$$

In (2.3) the angle brackets denote an ensemble average, $k$ is Boltzmann's constant, $T$ is the absolute temperature, and $\delta(t)$ is the delta function. The amplitude of the correlation between the Brownian forces at time 0 and at time $t$ results from the fluctuation-dissipation theorem for the $N$-body system.

The evolution equation for the particles is obtained by integrating (2.1) over a time step $\Delta t$ that is large compared with $\tau$, the inertial relaxation time $(\tau=m / 6 \pi \eta a)$, but small compared with the time over which the configuration changes. A second integration in time produces the evolution equation for the particle positions (both translational and orientational) with error of $o(\Delta t)$ :

$$
\left.\begin{array}{c}
\Delta \boldsymbol{x}=\operatorname{Pe}\left\{\langle\boldsymbol{U}\rangle+\boldsymbol{R}_{F U}^{-1} \cdot\left[\boldsymbol{R}_{F E}:\langle\hat{\boldsymbol{E}}\rangle+\dot{\gamma}^{*-1} \boldsymbol{F}^{P}\right]\right\} \Delta t+\nabla \cdot \boldsymbol{R}_{F U}^{-1} \Delta t+\boldsymbol{X}(\Delta t), \\
\langle\boldsymbol{X}\rangle=0 \quad \text { and } \quad\langle\boldsymbol{X}(\Delta t) \boldsymbol{X}(\Delta t)\rangle=2 \boldsymbol{R}_{F U}^{-1} \Delta t .
\end{array}\right\}
$$

Here, $\Delta x$ is the change in particle position during the time step $\Delta t$, and $X(\Delta t)$ is a random displacement due to Brownian motion that has zero mean and covariance given by the inverse of the resistance tensor. In (2.4) $x$ has been non-dimensionalized by the characteristic particle size $a$; the time by the diffusive time scale $a^{2} / D$, where $D=k T / 6 \pi \eta a$ is the diffusion coefficient of a single isolated particle; the rate-ofstrain tensor $\langle\hat{\boldsymbol{E}}\rangle$ by $\dot{\gamma}$, where $\dot{\gamma}=|\langle\boldsymbol{\Gamma}\rangle|$ is the magnitude of the shear rate; the shear forces by $6 \pi \eta a^{2} \dot{\gamma}$; and the interparticle forces by their magnitude $\left|F^{P}\right|$. The Péclet number, $P e=\dot{\gamma} a^{2} / D=6 \pi \eta a^{3} \dot{\gamma} / k T$, measures the relative importance of shear and Brownian forces, and $\dot{\gamma}^{*}=6 \pi \eta a^{2} \dot{\gamma} /\left|\boldsymbol{F}^{P}\right|$ is a non-dimensional shear rate giving the relative importance of shear and interparticle or externally imposed forces. (Other non-dimensional parameters setting the range, as opposed to the amplitude, of the interparticle force may also be present.)

The evolution equation (2.4) shows that the suspension's behaviour depends on the dimensionless parameters: $P e, \dot{\gamma}^{*}$ and $\phi$, the volume fraction of particles. No restriction has been made to particles of identical size or shape; they need not be spherical, and if not, other dimensionless parameters characterizing their shape would be present.

The macroscopic properties are found from appropriate definitions and averages over particles and over time in a dynamic simulation. Here we shall be primarily interested in diffusion and rheology. Several 'particle diffusivities' may be defined. The short-time self-diffusivity $\boldsymbol{D}_{0}^{s}$, which measures the average instantaneous mobility of a particle, is given by an average over all configurations: $\boldsymbol{D}_{0}^{s}=\left\langle\boldsymbol{D}_{i i}\right\rangle$, where the subscript $i i$ (no sum on $i$ ) indicates that only the diagonal or self terms are included in the sum. The $N$-particle diffusion tensor $\boldsymbol{D}$ is given by the Stokes-Einstein relation:

$$
\boldsymbol{D} \equiv k T \boldsymbol{R}_{F U}^{-1} \text {. }
$$

The long-time self-diffusivity $\boldsymbol{D}_{\infty}^{s}$, which measures the ability of a particle to wander far from its starting point, is defined as the limit as time approaches infinity of one half of the time rate of change of the mean-square position of a particle:

$$
\boldsymbol{D}_{\infty}^{s}=\lim _{t \rightarrow \infty} \frac{1}{2} \frac{\mathrm{d}}{\mathrm{d} t}\left\langle(\boldsymbol{x}-\langle\boldsymbol{x}\rangle)^{2}\right\rangle .
$$


Both diffusivities are accessible by light scattering in tracer-diffusion experiments of equilibrium suspensions, since they are the short- and long-wavelength scattering limits, respectively (Rallison \& Hinch 1986; van Megen, Underwood \& Snook 1986; Pusey 1991; Brady 1994). Intermediate-time definitions are not possible, as the motion of a particle is not, in general, diffusive except at short and long times.

For rheology, the bulk stress $\langle\boldsymbol{\Sigma}\rangle$ is needed. This is defined as an average over the volume $V$ containing the $N$ particles and is given by

$$
\langle\boldsymbol{\Sigma}\rangle=-\langle p\rangle \boldsymbol{I}+2 \eta\langle\boldsymbol{E}\rangle+\left\langle\boldsymbol{\Sigma}_{p}\right\rangle,
$$

where $\langle p\rangle$ is a constant setting the level of the pressure in the incompressible medium, and $2 \eta\langle E\rangle$ is the deviatoric stress contribution from the fluid. The particle contribution to the stress $\left\langle\Sigma_{p}\right\rangle$ is given by

$$
\left\langle\Sigma_{p}\right\rangle=-n k T I+n\left\{\left\langle\mathbf{S}^{H}\right\rangle+\left\langle\boldsymbol{S}^{P}\right\rangle+\left\langle\mathbf{S}^{B}\right\rangle\right\} .
$$

Here, $-n k T I$ is the isotropic stress associated with the thermal energy of the Brownian particles, $I$ is the isotropic tensor, and $n$ is the number density of particles. The particles make three contributions to the bulk stress: $(a)$ a mechanical or contract stress transmitted by the fluid due to the shear flow, $\left\langle\boldsymbol{S}^{H}\right\rangle ;(b)$ a stress due to the interparticle forces, $\left\langle\boldsymbol{S}^{P}\right\rangle$; and $(c)$ a direct contribution from Brownian motion, $\left\langle\boldsymbol{S}^{B}\right\rangle$. Note that the contributions to the bulk stress parallel the forces in the Langevin equation (2.1) and are given by

$$
\begin{aligned}
& \left\langle\boldsymbol{S}^{H}\right\rangle=-\left\langle\boldsymbol{R}_{S U} \cdot \boldsymbol{R}_{F U}^{-1} \cdot \boldsymbol{R}_{F E}-\boldsymbol{R}_{S E}\right\rangle:\langle\boldsymbol{E}\rangle, \\
& \left\langle\mathbf{S}^{P}\right\rangle=-\left\langle\left(\boldsymbol{R}_{S U} \cdot \boldsymbol{R}_{F U}^{-1}+\boldsymbol{x} \boldsymbol{I}\right) \cdot \boldsymbol{F}^{P}\right\rangle, \\
& \left\langle\boldsymbol{S}^{B}\right\rangle=-k T\left\langle\nabla \cdot\left(\boldsymbol{R}_{S U} \cdot \boldsymbol{R}_{F U}^{-1}\right)\right\rangle .
\end{aligned}
$$

The configuration-dependent resistance tensors $\boldsymbol{R}_{S U}(\boldsymbol{x})$ and $\boldsymbol{R}_{S E}(\boldsymbol{x})$ are similar to $\boldsymbol{R}_{F U}$ and $\boldsymbol{R}_{F E}$ and relate the particle 'stresslet' $\boldsymbol{S}$ to the particle velocities and to the imposed rate of strain, respectively. The stresslet is the symmetric (but not traceless) first moment of the force distribution integrated over the particle surface.

The evolution equation (2.4) and the macroscopic properties (2.6)-(2.9) are the heart of the dynamic simulation. They are an exact description for $N$ particles of arbitrary size and shape suspended in a volume $V$ interacting through hydrodynamic, interparticle or external, and Brownian forces. Given an initial configuration, (2.4) is integrated in time to follow the dynamic evolution of the suspension microstructure, and (2.6)-(2.9) evaluated to determine the diffusive and rheological behaviour. In addition to these macroscopic properties, a large number of particle distribution functions (e.g. $g(\mathbf{r})$, etc.) can be determined.

In order to make use of the evolution equation and calculate macroscopic properties, the hydrodynamic resistance tensors $\boldsymbol{R}_{F U}, \boldsymbol{R}_{F E}$, etc. must be determined. A method that accurately and efficiently accounts for the near-field lubrication effects and the dominant many-body interactions has been developed (Durlofsky et al. 1987; Brady et al. 1988). Lubrication forces, as the name implies, result from the thin layer of viscous fluid separating particle surfaces and result in, among other effects, the relative motion of two particles approaching zero as their surfaces touch.

The resistance tensors $\boldsymbol{R}_{F U}, \boldsymbol{R}_{F E}, \boldsymbol{R}_{S U}$ and $\boldsymbol{R}_{S E}$ can be written as part of a 'grand resistance' tensor $\mathscr{R}$ that relates the force/torque $(\boldsymbol{F})$ and stresslet $(\boldsymbol{S})$ exerted by the fluid on the particles to the particle velocities and the rate of strain. The corresponding inverse or 'grand mobility' tensor $\mathscr{M}$ is $\mathscr{M}=\mathscr{R}^{-1}$. The method proceeds by constructing an approximation to $\mathscr{M}$ by combining Faxén laws for particle velocities 
with a truncated multipole-moments representation of the particles. The grand mobility tensor, denoted $\mathscr{M}^{\infty}$, is then inverted to yield a far-field approximation to the grand resistance tensor. This many-body approximation to the resistance tensor lacks, however, lubrication. Lubrication would only be reproduced upon inversion of the mobility tensor if all multipole moments were included. Because of their short-range nature, lubrication forces are two-body interactions and are introduced in a pairwise additive fashion in the resistance tensor. Thus, the approximate grand resistance tensor that includes near-field lubrication and far-field many-body interactions is

$$
\mathscr{R}=\left(\mathscr{M}^{\infty}\right)^{-1}+\mathscr{R}_{l u b},
$$

where $\mathscr{R}_{l u b}$ stands for the near-field lubrication interactions. The grand resistance tensor is then partitioned and used in the evolution equation (2.4) and the macroscopic properties (2.6)-(2.9). This procedure captures both the near- and far-field physics and has given excellent results for all situations in which a comparison has been possible (Durlofsky et al. 1987; Brady et al. 1988; Phillips, Brady \& Bossis 1988).

The long-range $(1 / r)$ nature of the hydrodynamic interactions requires care in simulating infinite suspensions, i.e. letting $N \rightarrow \infty, V \rightarrow \infty$, keeping $n=N / V$ fixed. A simple summation of interactions results in badly divergent expressions. While there are several alternative ways to overcome this convergence problem, the method presented by O'Brien (1979) can conveniently be used in dynamic simulation. Used with periodic boundary conditions this method 'renormalizes' all divergent and conditionally convergent hydrodynamic interactions and accelerates the convergence of the interactions using the Ewald summation technique. As a final note, Brownian motion requires the evaluation of the divergence of the inverse of the resistance tensor both in the displacement, $\nabla \cdot \boldsymbol{R}_{F U}^{-1}$, and the stress, $\nabla \cdot \boldsymbol{R}_{S U} \cdot \boldsymbol{R}_{F U}^{-1}$. These divergences were calculated analytically and implemented numerically using the identity $\nabla \cdot \boldsymbol{R}_{F U}^{-1}=$ $-\boldsymbol{R}_{F U}^{-1} \cdot \nabla \boldsymbol{R}_{F U}: \boldsymbol{R}_{F U}^{-1}$. Alternately, one can return to the Langevin equation and integrate with a two-point method to numerically compute the derivatives (Grassia, Hinch \& Nitsche 1995).

As currently implemented, calculation of the hydrodynamic interactions requires $O\left(N^{3}\right)$ operations. Filling the grand mobility tensor and performing the Ewald summations are $O\left(N^{2}\right)$, while inverting this mobility tensor requires $O\left((11 N)^{3}\right)$ operations; the 11 degrees of freedom correspond to three translational, three rotational and five components of the stresslet. (The trace of the stresslet gives the suspension pressure, but does not couple to the particle dynamics in the incompressible medium.) Calculation of the resistance invert $\boldsymbol{R}_{F U}^{-1}$ as well as its divergence are $O\left((6 N)^{3}\right)$, as only six degrees of freedom are required. Iterative methods along with schemes to produce sparse matrices (Brady \& Bossis 1988) could be used to reduce the computational burden to $O(N)$, but are unnecessary for the relatively small systems sizes we have employed. Since Brownian motion introduces a random displacement at each time step, a simple first-order explicit time integration is employed. Without Brownian motion a higher-order-accurate time integration is needed to faithfully reproduce particle trajectories; a first-order scheme produces aphysical behaviour such as loss of flowreversal symmetry in the pure hydrodynamic limit. Despite the singular lubrication forces a small amount $\left(-10^{-2} a<r-2 a<0\right)$ of particle overlap may occur because of the finite time step. When overlap occurs we set the interactions at a non-dimensional surface-surface separation of $10^{-8}$ and allow the integration to proceed. This method preserves the symmetry of particle trajectories in the pure hydrodynamic limit (Bossis \& Brady 1987) and produces the correct equilibrium structure in the pure Brownian limit (cf. figure 1). A multiple time-stepping scheme is also used since the mobility 


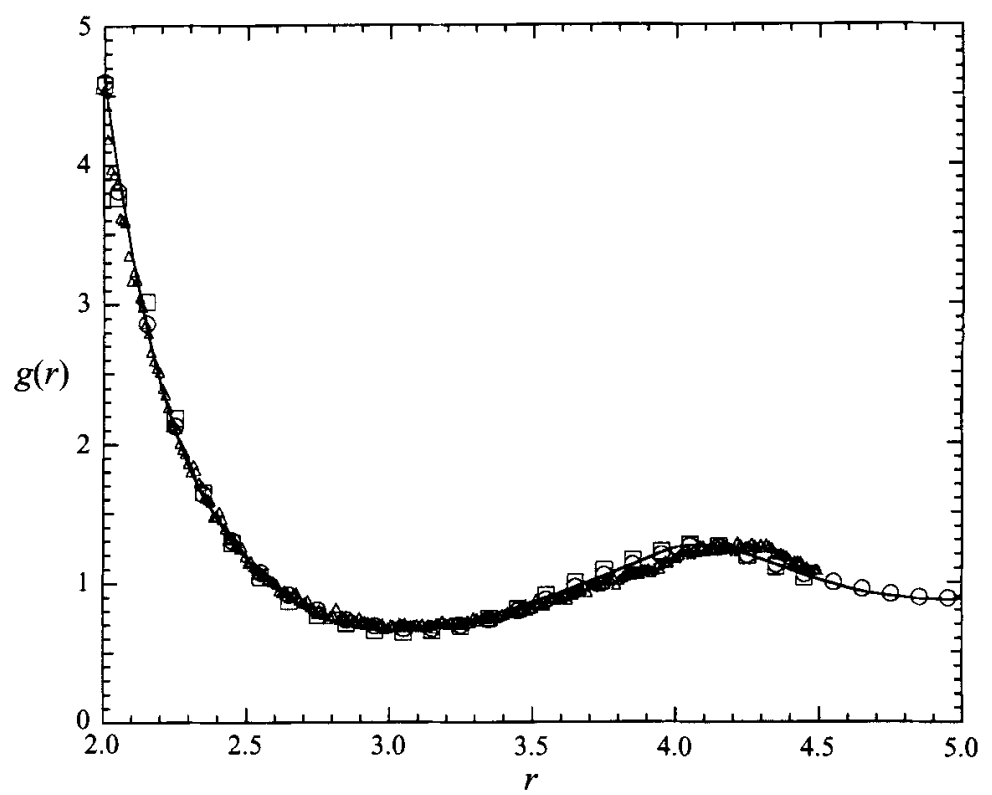

FIGURE 1. Comparison of the hard-sphere radial-distribution function at $\phi=0.45$ : solid curve, Monte Carlo; symbols, Stokesian Dynamics at $P e \equiv 0: \triangle, N=27 ; \square, N=81 ; \bigcirc, N=123$. For the smallest system the influence of the periodic simulation cell can be seen in the shift in the second nearest-neighbour peak.

interactions vary on the scale of the particle size, while the lubrication interactions vary on the scale of the particle surface separation. The time step is governed by accurately resolving the close particle encounters and the mobility interactions are computed less frequently so that the computer time required in the two sections is roughly the same. As an example of the computational times, 40000 time steps for $N=27$ require approximately 4.3 hours on an IBM RISC/6000 model 530 (rated at 10 MFLOPS) workstation.

The simulation results to be presented in the next section are for a monodisperse suspension of Brownian hard spheres. For particles interacting as hard spheres the interparticle force is identically zero, $\boldsymbol{F}^{P} \equiv 0$. The no-slip hydrodynamic boundary condition at particle surfaces guarantees that the particles behave as hard spheres (Brady 1993a). This can be appreciated by noting that an interparticle force of hard-sphere type at contact $r=2 a$ between two particles, $\boldsymbol{F}^{P}=\frac{1}{2} k T \hat{r} \delta(r-2 a)$, has no dynamical consequence. Since the relative mobility of two particles vanishes at contact as $r-2 a$ due to the lubrication interactions, the relative velocity caused by a hard-sphere force is proportional to $(r-2 a) \delta(r-2 a)$ and vanishes. Simulations with $\boldsymbol{F}^{P} \equiv 0$ at $P e \equiv 0$ as illustrated in figure 1 reproduce precisely the expected hard-sphere behaviour. Similarly, the stress contribution from hard-sphere forces at contact is zero $\left(\boldsymbol{S}^{P} \equiv 0\right)$. Thus, for a hard-sphere suspension the number of parameters reduces to two: the Péclet number $P e$ and the volume fraction $\phi$.

\section{Results}

In order to further reduce the number of parameters we shall present results at a single volume fraction of $\phi=0.45$. This volume fraction was chosen because it is sufficiently high that interesting rheological and structural behaviour is observed 
as the Péclet number is varied; it is also well below the equilibrium volume fraction $\phi=0.494$ at which the hard-sphere suspension undergoes a phase transition from a disordered liquid to a solid. Results from simulations at higher and lower volume fractions are available elsewhere (Phung 1993; Phung, Brady \& Bossis 1996a,b).

A large number of simulations were performed for Péclet numbers ranging from 0 to $10^{5}$ and the number of particles $N$ in the unit cell varied from 27 to 123. Information from these simulations is summarized in tables 1 and 2. For $P e<1$ time was nondimensionalized with $a^{2} / D$ as in (2.4), while for $P e>1$ time was non-dimensionalized with the inverse shear rate $1 / \dot{\gamma}$. Also, many long runs were divided up into statistically independent subintervals in order to determine the statistical variation in properties. All runs for all Péclet numbers, unless specifically noted, were started at the equilibrium random hard-sphere configuration generated by a Monte Carlo procedure. In each case the first 5000 to 10000 time steps were ignored when computing average properties. The properties reported are averages over all particles and over time. All simulations are for simple shear flow with the flow, velocity-gradient, and vorticity directions along the three axes $(x, y, z)$ of the cubic unit cell. Owing to space limitations only select average results are shown in the tables. Additional data and further details of the simulation method and data analysis are in Phung (1993).

We can anticipate three regimes of behaviour as the shear rate or Péclet number is varied. At low Péclet number Brownian forces dominate and the flow acts as a perturbation (regular) to the equilibrium behaviour. At intermediate Péclet numbers Brownian and hydrodynamic forces balance to set the structure and determine properties. And at high Péclet numbers hydrodynamic forces dominate with Brownian motion representing a perturbation, although not necessarily weak (singular). The presentation below is divided into three parts: rheology, diffusive behaviour and structure.

\subsection{Rheology}

The suspension viscosity relative to that of the solvent is defined for simple shear flow from the $(x, y)$ components of the bulk stress and rate of strain:

$$
\eta_{r} \equiv \frac{\Sigma_{x y}}{2 \eta E_{x y}} .
$$

The individual Brownian $\left(\mathbf{S}^{B}\right)$ and hydrodynamic $\left(\boldsymbol{S}^{H}\right)$ contributions to the relative viscosity are denoted as $\eta_{r}^{B}$ and $\eta_{r}^{H}$, respectively; hence

$$
\eta_{r}=1+\eta_{r}^{B}+\eta_{r}^{H},
$$

where the 1 is the solvent contribution. At infinite dilution an isolated particle gives the Einstein viscosity $\eta_{r}^{H}=\frac{5}{2} \phi$; for spherical particles the Brownian viscosity is $O\left(\phi^{2}\right)$.

In figure 2 the results from table 1 for $N=27$ for the individual viscosity contributions are shown as a function of the Péclet number. The Brownian viscosity shear thins, becoming insignificant compared to the hydrodynamic viscosity for $P e>10$. The hydrodynamic viscosity remains roughly constant throughout the shear thinning process and then increases for $P e>100$. The total viscosity thus shear thins and shear thickens. This finding is in complete agreement with the monolayer simulations we reported earlier (Bossis \& Brady 1989). The constancy of the hydrodynamic viscosity and the shear thinning of the Brownian viscosity has been shown in the recent stress jump experiments of MacKay \& Kaffashi (1995).

The shear thinning can be understood as follows. The Brownian stress arises from the flow-induced deformation of the equilibrium structure; particles diffuse against the flow towards their unstressed configuration and the resultant stress is directly 


\begin{tabular}{|c|c|c|c|c|c|c|c|}
\hline $\mathrm{Pe}$ & $N$ & $\Delta t$ & NSTEPS & $\eta_{r}^{B}$ & $\eta_{r}^{H}$ & $N_{1} / \eta \dot{\gamma}$ & $N_{2} / \eta \dot{\gamma}$ \\
\hline 0.01 & 27 & $5 \times 10^{-4}$ & 1000000 & $9.0 \pm 9.1$ & $4.61 \pm 0.01$ & $0.21 \pm 0.15$ & $-0.10 \pm 0.05$ \\
\hline 0.10 & 27 & $10^{-3}$ & 500000 & $7.1 \pm 0.6$ & $4.57 \pm 0.04$ & $0.62 \pm 0.40$ & $-0.25 \pm 0.33$ \\
\hline 0.43 & 27 & $10^{-3}$ & 50000 & $6.29 \pm 0.06$ & $4.71 \pm 0.01$ & $0.67 \pm 0.04$ & $-0.94 \pm 0.03$ \\
\hline 1.00 & 27 & $10^{-3}$ & 100000 & $3.74 \pm 0.02$ & $4.80 \pm 0.01$ & $0.46 \pm 0.09$ & $-1.87 \pm 0.06$ \\
\hline 3.00 & 27 & $10^{-3}$ & 150000 & $1.46 \pm 0.07$ & $4.51 \pm 0.08$ & $-0.46 \pm 0.13$ & $-0.86 \pm 0.18$ \\
\hline 5.00 & 27 & $10^{-3}$ & 50000 & $1.32 \pm 0.02$ & $4.47 \pm 0.02$ & $-0.29 \pm 0.05$ & $-0.63 \pm 0.05$ \\
\hline 7.00 & 27 & $10^{-3}$ & 50000 & $1.29 \pm 0.07$ & $4.32 \pm 0.12$ & $0.13 \pm 0.04$ & $-0.76 \pm 0.02$ \\
\hline 10.00 & 27 & $10^{-3}$ & 100000 & $1.08 \pm 0.03$ & $4.51 \pm 0.06$ & $-0.15 \pm 0.14$ & $-0.58 \pm 0.11$ \\
\hline 20.00 & 27 & $10^{-3}$ & 60000 & $0.40 \pm 0.04$ & $6.05 \pm 016$ & $0.08 \pm 0.15$ & $-1.10 \pm 0.13$ \\
\hline $10^{2}$ & 27 & $10^{-3}$ & 100000 & $0.184 \pm 0.005$ & $6.59 \pm 0.05$ & $-0.70 \pm 0.18$ & $-1.42 \pm 0.09$ \\
\hline $2 \times 10^{2}$ & 27 & $10^{-3}$ & 60000 & $0.108 \pm 0.002$ & $7.10 \pm 0.05$ & $-1.60 \pm 0.10$ & $-0.94 \pm 0.07$ \\
\hline $10^{3}$ & 27 & $10^{-3}$ & 100000 & $0.030 \pm 0.001$ & $9.22 \pm 0.10$ & $-1.65 \pm 0.07$ & $-2.16 \pm 0.05$ \\
\hline $10^{4}$ & 27 & $10^{-3}$ & 70000 & $0.003 \pm 0.00$ & $10.89 \pm 0.09$ & $-1.72 \pm 0.09$ & $-2.45 \pm 0.14$ \\
\hline $10^{5}$ & 27 & $10^{-3}$ & 100000 & $2.7 \times 10^{-4}$ & $13.9 \pm 1.7$ & $3.8 \pm 2.2$ & $-2.1 \pm 1.2$ \\
\hline$* 10.00$ & 27 & $10^{-3}$ & 100000 & $0.80 \pm 0.02$ & $4.64 \pm 0.05$ & $0.14 \pm 0.11$ & $0.90 \pm 0.14$ \\
\hline 0.01 & 63 & $5 \times 10^{-4}$ & 80000 & $10.2 \pm 8.2$ & $4.42 \pm 0.01$ & & \\
\hline 0.43 & 63 & $10^{-3}$ & 40000 & $6.48 \pm 0.13$ & $4.78 \pm 0.02$ & $0.37 \pm 0.04$ & $-0.58 \pm 0.04$ \\
\hline 10.00 & 63 & $10^{-3}$ & 100000 & $1.17 \pm 0.00$ & $4.60 \pm 0.07$ & $-0.38 \pm 0.04$ & $-0.82 \pm 0.05$ \\
\hline 10.00 & 64 & $10^{-3}$ & 100000 & $1.18 \pm 0.01$ & $4.36 \pm 0.01$ & $-0.09 \pm 0.03$ & $-0.60 \pm 0.03$ \\
\hline $10^{4}$ & 63 & $10^{-3}$ & 50000 & $0 . \overline{003}$ & $10.06 \pm 0.20$ & $-1.50 \pm 0.16$ & $-2.22 \pm 0.25$ \\
\hline 0.01 & 81 & $5 \times 10^{-4}$ & 120000 & $9.9 \pm 3.8$ & $4.78 \pm 0.01$ & & \\
\hline 0.43 & 81 & $10^{-3}$ & 60000 & $6.63 \pm 0.05$ & $4.79 \pm 0.00$ & $0.75 \pm 0.08$ & $-0.70 \pm 0.02$ \\
\hline 10.00 & 81 & $10^{-3}$ & 60000 & $1.29 \pm 0.02$ & $4.59 \pm 0.04$ & $1.15 \pm 0.05$ & $-0.72 \pm 0.03$ \\
\hline $10^{4}$ & 81 & $10^{-3}$ & 55000 & $0 . \overline{003}$ & $10.54 \pm 0.03$ & $-1.59 \pm 0.08$ & $-2.24 \pm 0.02$ \\
\hline 0.43 & 123 & $5 \times 10^{-4}$ & 80000 & 5.454 & 4.65 & 0.45 & -0.56 \\
\hline 10.00 & 123 & $10^{-3}$ & 50000 & 1.14 & 4.33 & 0.04 & -1.13 \\
\hline $10^{3}$ & 123 & $10^{-3}$ & 40000 & 0.025 & 8.59 & 0.005 & -1.4 \\
\hline 0.01 & $25,2 \mathrm{D}$ & $5 \times 10^{-4}$ & 700000 & $9.2 \pm 6.6$ & $4.82 \pm 0.04$ & $0.75 \pm 0.38$ & \\
\hline 10.00 & $25,2 \mathrm{D}$ & $10^{-3}$ & 350000 & $1.01 \pm 0.11$ & $4.87 \pm 0.43$ & $0.11 \pm 0.45$ & \\
\hline $10^{3}$ & $25,2 \mathrm{D}$ & $10^{-3}$ & 240000 & $0.025 \pm 0.002$ & $9.46 \pm 0.34$ & $-1.75 \pm 0.43$ & \\
\hline 10.00 & $25,2 \mathrm{D}$ & $10^{-3}$ & 300000 & $1.07 \pm 0.10$ & $4.70 \pm 0.40$ & $0.06 \pm 0.36$ & \\
\hline
\end{tabular}

TABLE 1. Simulations data. Column 1 is the Péclet number and column 2 is the number of particles. Columns 3 and 4 are the time step and the total number of time steps. Columns 5 and 6 give the Brownian and hydrodynamic contributions to the shear viscosity (normalized by the solvent viscosity). And columns 7 and 8 give the first and second normal stress differences. Initial particle configurations of all the runs are random except the case with $P e=* 10$. In this run the initial particle placement is obtained from the end of the prior run with $P e=10$ and a new shear direction along the $y$-axis was used. The error estimates were determined by dividing a simulation run into statistically independent subintervals (5-10) and comparing the averages for each interval (see Phung 1993). The last four entries correspond to monolayer simulations performed in the sequence indicated $P e=0.01 \rightarrow 10 \rightarrow 10^{3} \rightarrow 10$. There are no error bars on the simulations with $N=123$ because only one run was made. At $P e=0.01$ normal stress differences could not be determined because the runs were too short to obtain adequate statistics.

proportional to the deformation. For small Péclet numbers the deformation is linear in the shear rate and proportional to $P e$. Since the stress is normalized hydrodynamically by $\eta \dot{\gamma}$, the Brownian contribution to the stress scales as the 'deformation' $P e$; hence, $S^{B} \sim O(1)$ as $P e \rightarrow 0$. From figure 2 we see that the Brownian viscosity appears to approach a constant as $P e \rightarrow 0$, although $P e=0.01$ may not be small enough to have reached the asymptote. In order for the Brownian stress to remain constant as the 


\begin{tabular}{|c|c|c|c|c|c|c|}
\hline$P e$ & $N$ & $\langle g(2)\rangle_{\Omega}$ & $D_{0}^{\mathrm{s}}$ & $D_{0}^{r}$ & $D_{y y}$ & $D_{z z}$ \\
\hline 0.01 & 27 & 4.20 & 0.172 & 0.553 & $0.068 \pm 0.003$ & $0.058 \pm 0.008$ \\
\hline 0.10 & 27 & 4.44 & 0.177 & 0.555 & $0.076 \pm 0.015$ & $0.065 \pm 0.005$ \\
\hline 0.43 & 27 & 4.94 & 0.170 & 0.544 & $0.105 \pm 0.005$ & $0.079 \pm 0.010$ \\
\hline 1.00 & 27 & 5.60 & 0.165 & 0.533 & $0.148 \pm 0.033$ & $0.179 \pm 0.035$ \\
\hline 3.00 & 27 & 0.95 & 0.180 & 0.563 & $0.16 \pm 0.02$ & $0.16 \pm 0.03$ \\
\hline 5.00 & 27 & 0.59 & 0.175 & 0.552 & $0.1 \pm 0.01$ & $0.1 \pm 0.01$ \\
\hline 7.00 & 27 & 0.73 & 0.162 & 0.528 & $0.02 \pm 0.01$ & $0.10 \pm 0.01$ \\
\hline 10.00 & 27 & 0.98 & 0.159 & 0.521 & $0.003 \pm 0.000$ & $0.04 \pm 0.000$ \\
\hline 20.00 & 27 & 7.26 & 0.146 & 0.492 & $0.36 \pm 0.02$ & $0.4 \pm 0.04$ \\
\hline $10^{2}$ & 27 & 32.6 & 0.114 & 0.412 & $2.9 \pm 0.3$ & $2.5 \pm 0.2$ \\
\hline $2 \times 10^{2}$ & 27 & 40.4 & 0.105 & 0.385 & $5.6 \pm 0.6$ & $5.4 \pm 0.8$ \\
\hline $10^{3}$ & 27 & 48.9 & 0.091 & 0.330 & $39 \pm 4$ & $46 \pm 3$ \\
\hline $10^{4}$ & 27 & 56.6 & 0.070 & 0.251 & $580 \pm 16$ & $540 \pm 90$ \\
\hline $10^{5}$ & 27 & 66.0 & 0.055 & 0.210 & & \\
\hline 0.01 & 63 & 4.51 & 0.204 & 0.558 & & \\
\hline 0.43 & 63 & 4.46 & 0.184 & 0.541 & & \\
\hline 10.00 & 63 & 1.35 & 0.177 & 0.524 & & \\
\hline 10.00 & 64 & 1.35 & 0.177 & 0.526 & & \\
\hline $10^{4}$ & 63 & 54.4 & 0.083 & 0.282 & & \\
\hline 0.01 & 81 & 4.53 & 0.191 & 0.549 & & \\
\hline 0.43 & 81 & 5.05 & 0.189 & 0.541 & & \\
\hline 10.00 & 81 & 1.30 & 0.195 & 0.555 & & \\
\hline $10^{4}$ & 81 & 56.2 & 0.086 & 0.277 & & \\
\hline 0.43 & 123 & 4.94 & 0.204 & 0.551 & & \\
\hline 10.00 & 123 & 0.84 & 0.185 & 0.521 & & \\
\hline $10^{3}$ & 123 & 47.9 & 0.110 & 0.341 & & \\
\hline 0.01 & $25,2 \mathrm{D}$ & & 0.333 & 0.582 & & \\
\hline 10.00 & $25,2 \mathrm{D}$ & & 0.333 & 0.582 & & \\
\hline $10^{3}$ & $25,2 \mathrm{D}$ & & 0.214 & 0.316 & & \\
\hline 10.00 & $25,2 \mathrm{D}$ & & 0.336 & 0.589 & & \\
\hline
\end{tabular}

TABLE 2. Same runs as in table 1 but showing the angularly averaged pair-distribution function at contact and the self-diffusivities normalized by the isolated particle Brownian diffusivities. The error on the short-time self-diffusivities is \pm 1 in the last digit. For $N>27$ no long-time diffusivities were obtained because the runs were too short to obtain adequate statistics.

Péclet number increases the structural deformation must increase linearly with $P e$. However, the Péclet number is also the ratio of the relaxation time due to diffusion $a^{2} / D$ to the time scale of the flow $1 / \dot{\gamma}$, and as the Péclet number increases the particle motion cannot keep up with the flow and the structural deformation saturates. Hence the Brownian stress decreases as $P e \rightarrow \infty$. Recent theoretical work (Morris \& Brady $1996 b$ ) predicts the Brownian stress to decay as $1 / P e$, which is in agreement with the results of the simulations (cf. table 1).

The hydrodynamic stress arises because the particles are rigid and do not deform as fluid elements. At $\phi=0.45$ the hydrodynamic viscosity varies little between a random and a regular array and is roughly the same for any 'well-dispersed' structure. Although the structure evolves quite significantly at low Péclet numbers (cf. figure 8), the suspension remains 'well-dispersed' and the hydrodynamic viscosity is constant. In simple shear flow particles are pushed together along the compressive axis of the flow, while the action of Brownian motion is to keep particles apart and well 


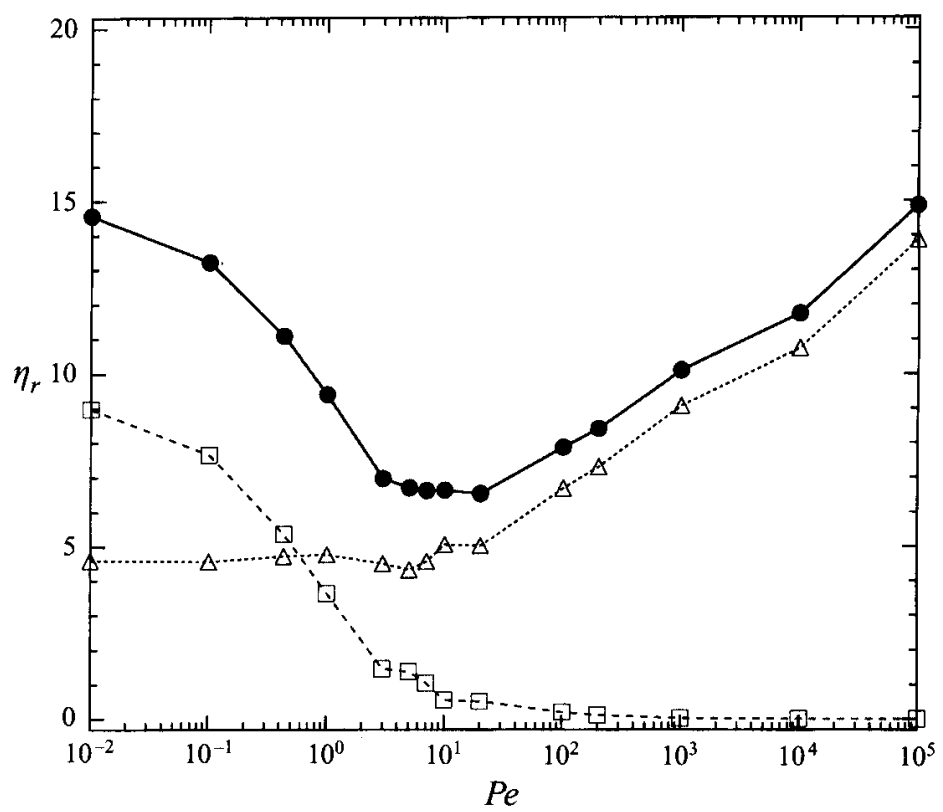

FIGURE 2. Péclet-number dependence of the relative viscosity of hard-sphere suspensions at $\phi=0.45$ determined by Stokesian Dynamics: total $(\bullet)$, hydrodynamic $(\triangle)$, and Brownian $(\square)$ viscosities.

dispersed. (In fact it is the $\nabla \cdot \boldsymbol{R}_{F U}^{-1}$ term in (2.4) that acts as a repulsive radial force and balances the hydrodynamic squeezing force along the compressive axis (Bossis \& Brady 1989).) At high Péclet numbers hydrodynamic forces dominate everywhere except in a thin $O\left(P e^{-1}\right)$ boundary layer adjacent to particle surfaces where there is a balance of hydrodynamic and Brownian forces (Morris \& Brady 1996b). Once the Péclet number exceeds $O(100)$ hydrodynamic forces are capable of pushing two particles close enough together for the strong lubrication forces to come into play; lubrication forces are singular near touching as $1 /(r-2 a)$, with this singularity being felt when $r-2 a<10^{-2} a$. As the Péclet number is increased particles are progressively 'stuck' together by the strong lubrication forces and form non-compact aggregates or clusters. As shown in our earlier work on monolayers (Bossis \& Brady 1989) the cluster size grows as the Péclet number increases. Associated with a growing cluster size is an increase in the contact value of the pair-distribution function reported in table 2. The increased contact value is also evident in the sharpening of the first nearest-neighbour peak in figure 11.

The fact that the clusters must be non-compact and that non-compact clusters lead to an increase in the viscosity can be shown by a simple scaling argument. All of the increase in the viscosity at high Péclet number comes from the hydrodynamic stress, and hydrodynamic stresses scale as the cube of the largest length of an object. If we have $N$ particles of size $a$ that are 'well-dispersed' the hydrodynamic stress will be proportional to $\mathrm{Na}^{3}$. How can we take the same $N$ particles and arrange them so as to increase the viscosity? If we formed one compact cluster of size $b$, then the stress would scale as $b^{3}$. But $b=N^{1 / 3} a$ for a compact cluster and the viscosity would be unchanged. (Note that the Einstein viscosity is independent of the size of the particles.) By forming non-compact clusters of size $\ell=N^{\alpha} a$, with $\alpha>1 / 3$, the viscosity will scale as $N^{3 \alpha} a^{3}$ and can be made arbitrary large as $N \rightarrow \infty$. Thus 


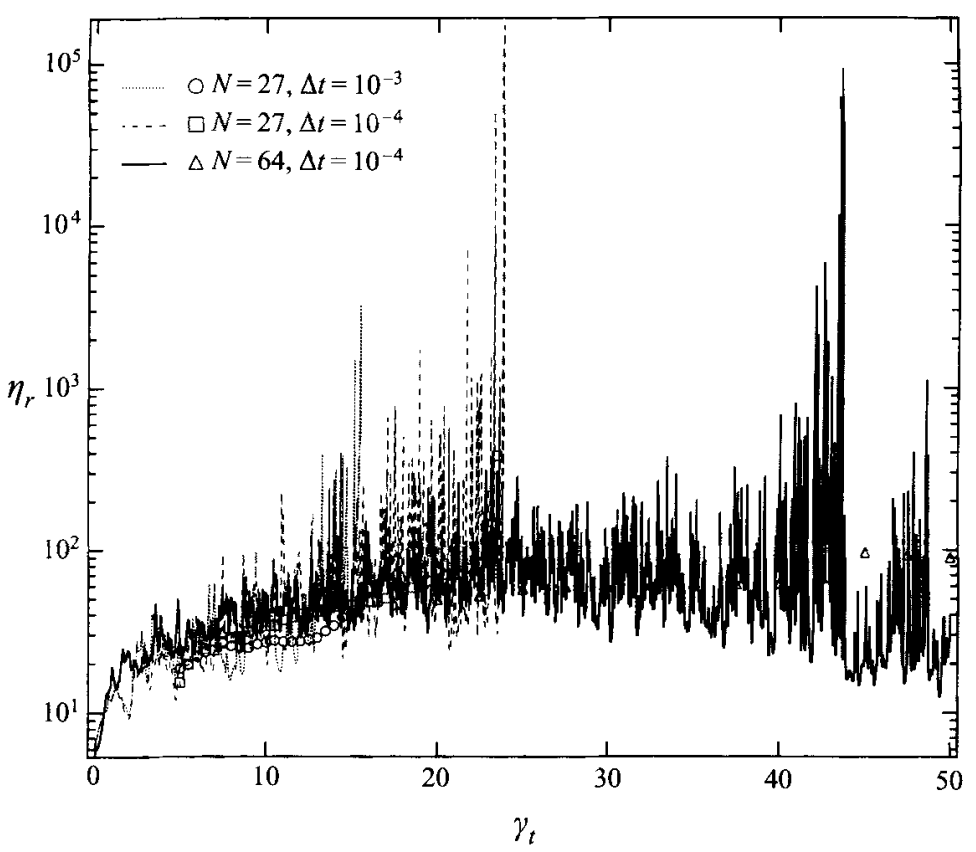

Figure 3. The suspension viscosity at $\phi=0.45$ for $N=27$ and 64 for various time steps as a function of time in the pure hydrodynamic limit, $P e^{-1} \equiv 0$. The symbols are the running average and the curves are the instantaneous value of the viscosity. A steady state was not achieved because large particle clusters formed with catastrophic shear thickening. Decreasing the time step or increasing the number of particles in the unit cell only delay the catastrophic failure. The run with $N=64$ failed just after the last time shown of 50 .

we have shear thickening due to the formation of large non-compact clusters as the hydrodynamic forces overcome Brownian motion. These clusters are dynamical with particles joining and leaving a cluster as the suspension is sheared.

Although the stress is purely hydrodynamic at large Péclet number, this does not imply that Brownian motion plays no role. The limit $P e \rightarrow \infty$ is singular and the residual effect of Brownian motion at particle contact limits the ultimate size of the clusters. The relative tangential motion of two particles is resisted by a weak logarithmic dependence on particle separation and the small amount of Brownian motion provides a means for two near-touching particles to move relative to one another and break the connectivity of the cluster, dramatically affecting the viscosity (Bossis, Meunier \& Brady 1991). Indeed, simulations in the pure hydrodynamic limit $\left(P e^{-1} \equiv 0\right)$ failed to reach a steady state. As shown in figure 3 the simulations would proceed in time with the viscosity slowly increasing until suddenly a large cell-spanning cluster would form jamming particles together, sending the viscosity to enormous values, and halting the integration. Reducing the time step allowed only a very small additional advance in time. Increasing the size of the unit cell delayed the onset of the catastrophic shear thickening but did not eliminate it. This is the reason the viscosity does not approach a high-Péclet-number asymptote in figure 2 .

This behaviour in the infinite-Péclet-number limit is 'percolation-like' in that at low enough volume fractions, which we have crudely estimated to be less than 0.4 , although clusters form they do not grow to 'infinite' extent and a steady state with a finite viscosity can be obtained. We have conducted simulations at Péclet 


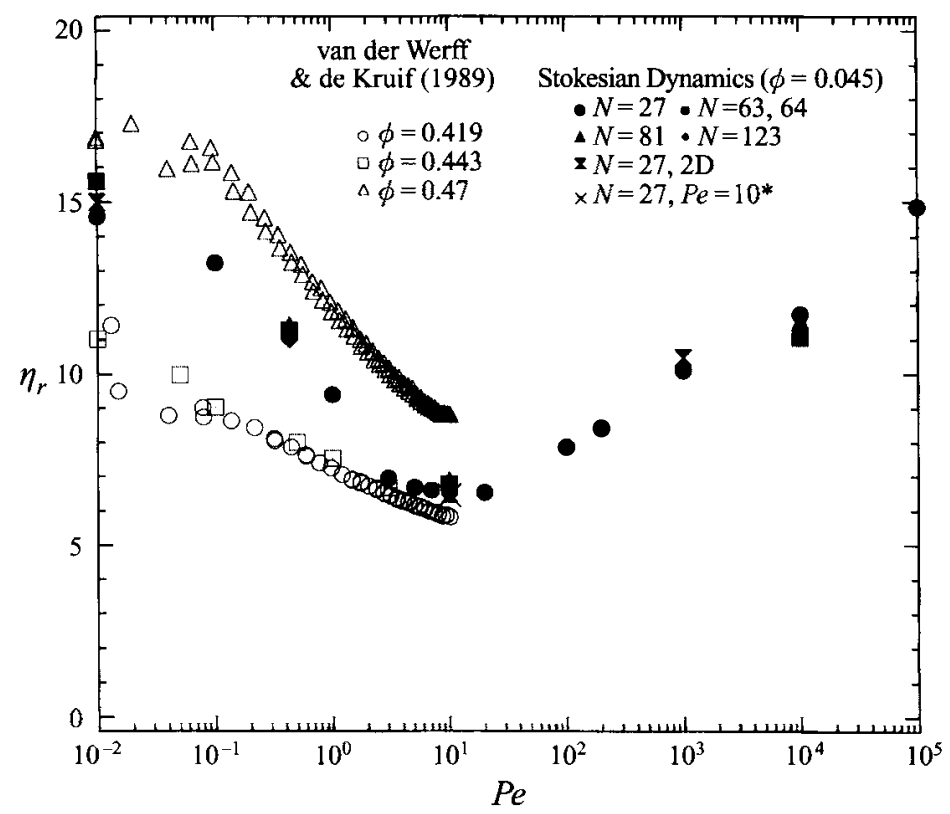

Figure 4. Comparison of the relative viscosity of hard-sphere suspensions determined by Stokesian Dynamics as a function of the Péclet number $P e$ (closed symbols, $N=27,63,64,81$, and 123; monolayer with $N=25$, and $\times$ denotes a change in the shear direction) with the experimental results (open symbols) of van der Werff \& de Kruif (1989).

numbers greater than $10^{5}$ and the viscosity reached a steady value, but we are concerned that the viscosity was limited by the clusters growing to the size of the simulation cell. Furthermore, the critical effect of Brownian motion is confined to the $O\left(P e^{-1}\right)$ boundary layer at particle surfaces and this boundary layer must be properly sampled in the simulations - an increasingly difficult task numerically - to capture the proper physics. This behaviour is also percolation-like in that any small effect such as Brownian motion, interparticle forces, or surface roughness can break particle clusters and result in a finite viscosity. This has profound implications for the interpretation of experimental viscosity measurements at high concentrations and shear rates because seemingly small factors can dramatically influence the results. It may also explain why measurements of viscosity at high concentrations in the pure hydrodynamic limit show a large amount of scatter (an order of magnitude) from one researcher to the next, although the reproducibility for an individual researcher was good (Thomas 1965). It also suggests that if short-range surface effects can be controlled and the Péclet number is made large enough the clusters should grow to the size of the experimental apparatus and the measured viscosity should depend on the size of the measuring cell.

In figure 4 we compare the simulation viscosities with the experiments of van der Werff \& de Kruif (1989) on monodisperse spherical silica particles that have been shown to behave to a very good approximation as hard spheres. We also see in this figure that the suspension viscosities determined by simulation are not sensitive to the number of particles in the unit cell: even monolayer simulations at an areal fraction of $0.675=3 / 2 \times 0.45$ give the same viscosity. The simulation and experimental viscosities are in good quantitative agreement considering the strong dependence of the viscosity on volume fraction at high volume fraction and the uncertainty in precisely determining the experimental volume fraction. The simulation and 


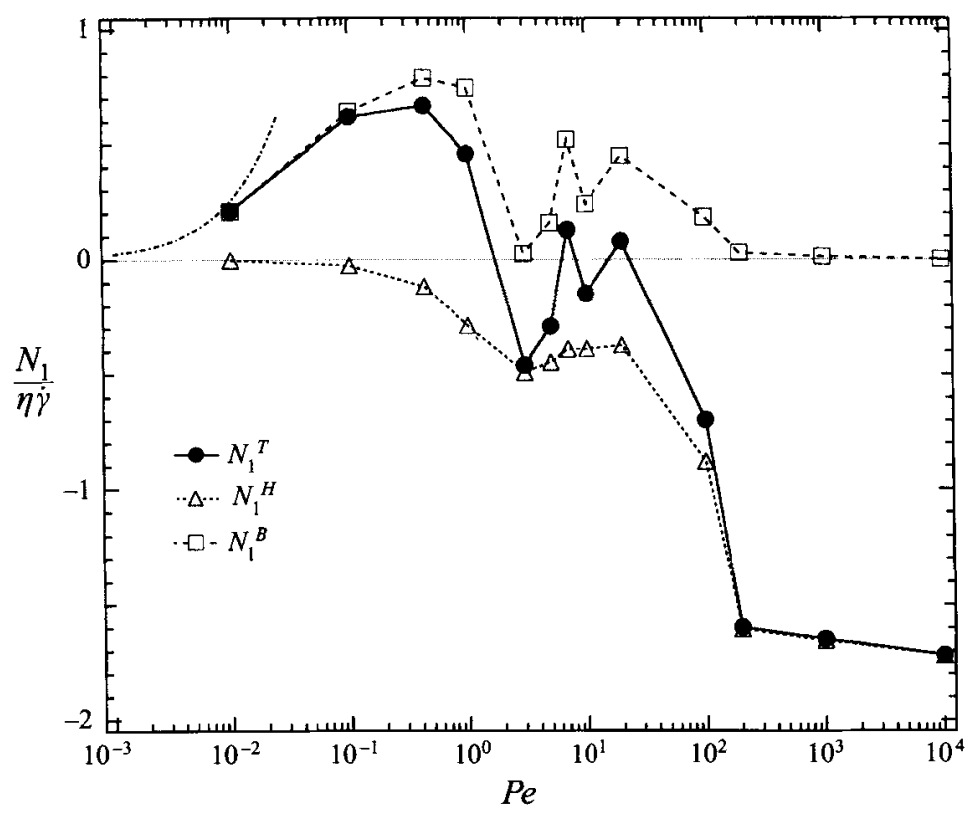

FIGURE 5. The hydrodynamic and Brownian contributions to the first normal stress difference for $N=27$ as a function of the Péclet number. The dashed-dotted curve is the scaling prediction of Brady \& Vicic (1995) as $P e \rightarrow 0$.

experimental results also agree well with the scaling theory of Brady (1993b) that predicts $\eta_{r} \sim 1.3\left(1-\phi / \phi_{m}\right)^{-2}$, as $\phi \rightarrow \phi_{m}$ for $P e \rightarrow 0$, where $\phi_{m} \approx 0.637$ is the volume fraction at random close packing. The experiments do not show shear thickening as the Péclet numbers were too low. Additional experiments by the same group up to $P e=1200$ did not display shear thickening. It may be that shear thickening occurs at a slightly higher Péclet number in these systems, possibly due to the fact that the silica particles are coated with short hydrocarbon chains and therefore are weakly repulsive when brought into near contact. However, shear thickening due solely to the growth of clusters as first predicted in our monolayer simulations has recently been seen experimentally (Dhaene, Mewis \& Fuller 1993; Bender \& Wagner 1995).

The first and second normal stress differences are defined by

$$
\begin{aligned}
& N_{1}=\left\langle\Sigma_{x x}\right\rangle-\left\langle\Sigma_{y y}\right\rangle, \\
& N_{2}=\left\langle\Sigma_{y y}\right\rangle-\left\langle\Sigma_{z z}\right\rangle,
\end{aligned}
$$

and the individual Brownian and hydrodynamic contributions are shown in figures 5 and 6. Determining the normal stress differences by simulation is quite difficult as they show much greater fluctuation from one time step to the next requiring very long runs to obtain a meaningful average. We do not report values for $P e=0.01$ for $N>27$ as the simulation runs are not long enough.

Unlike the shear viscosity, as $P e \rightarrow 0$ the normal stress differences vanish in accord with general flow-reversal symmetry requirements that $N_{1}, N_{2} \sim O(\eta \dot{\gamma} P e)$ as $P e \rightarrow 0$. In figures 5 and 6 we show the scaling predictions of Brady \& Vicic (1995): $N_{1} \sim 0.59\left(1-\phi / \phi_{m}\right)^{-3} P e$ and $N_{2} \sim-0.42\left(1-\phi / \phi_{m}\right)^{-3} \mathrm{Pe}$ as $\mathrm{Pe} \rightarrow 0$, and $\phi \rightarrow \phi_{m}$, which are in qualitative agreement with the simulations; the uncertainty in the data make a quantitative comparison impossible. Note that the hydrodynamic 


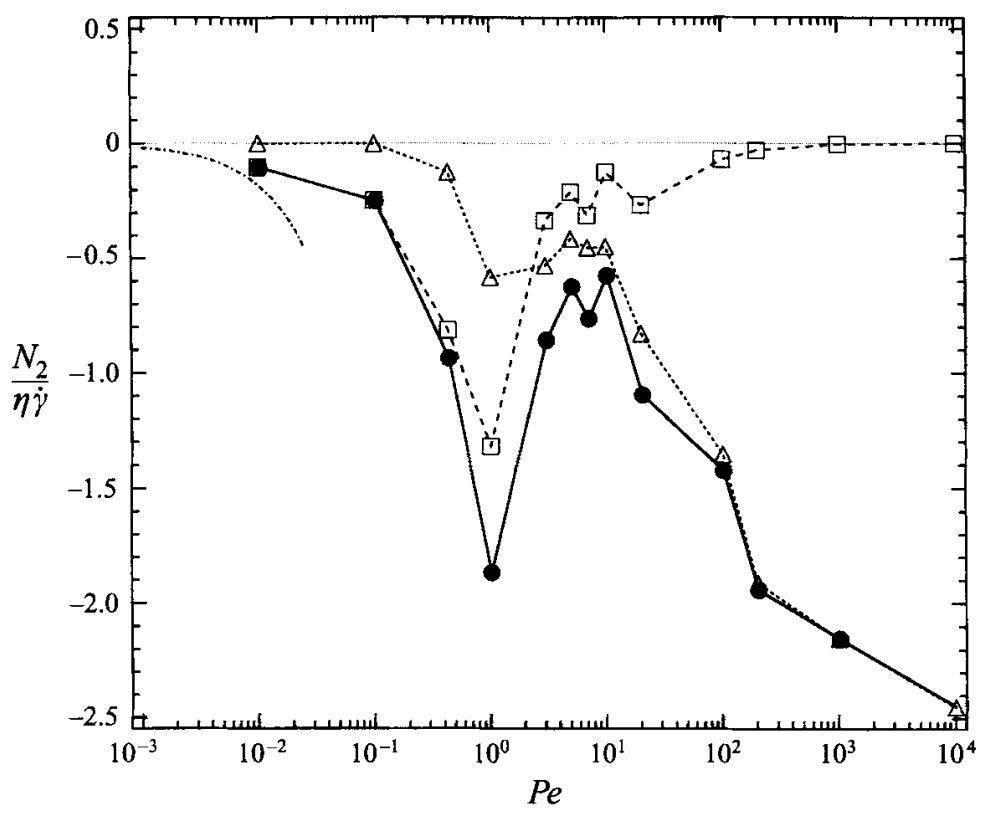

Figure 6. As figure 5 but for the second normal stress difference.

contributions are negative for all $P e$, whereas the Brownian contribution is positive for $N_{1}$ and negative for $N_{2}$.

A positive $N_{1}$ and negative $N_{2}$ for small $P e$ is common in polymeric systems. In polymeric systems $N_{2}$ is generally much smaller than $N_{1}$, while for colloids $N_{1}$ and $N_{2}$ are of the same magnitude. The similarity to polymeric systems can be readily appreciated by noting that the Brownian stress can be rewritten by introducing the $N$-particle probability distribution and integrating by parts to obtain

$$
\left\langle\mathbf{S}^{B}\right\rangle=-n^{2} k T 4 a^{3} \oint_{r=2} \hat{r} \hat{r} g(2) \mathrm{d} \Omega+n k T\left\langle\boldsymbol{R}_{S U} \cdot \boldsymbol{R}_{F U}^{-1} \cdot \nabla \ln P_{N}\right\rangle,
$$

where $g(2)$ is the pair-distribution function at contact, $\hat{\boldsymbol{r}}$ is a unit vector connecting two particles, and the integral is over the solid angle at contact. In concentrated suspensions the contact integral dominates (Brady 1993b). A stress proportional to an integral of $\hat{r} \hat{r} g(\boldsymbol{r})$ also occurs in polymer systems: the simplest Hookean-spring model of a polymer has a stress proportional to $\langle\hat{r} \hat{r}\rangle$, where $\hat{r}$ is the non-dimensional end-to-end vector describing the polymer molecule. This stress can either be viewed as an interparticle-force stress with a radial interparticle force, or as an entropic or Brownian stress as in colloidal suspensions. Indeed, the action of Brownian motion in colloidal dispersions in opposing relative motion of two particles along the compressive and extensional axes of the flow is the same as the entropic 'spring' force in polymeric systems.

At equilibrium $g(\boldsymbol{r})$ is a function of $r$ only and the stress is isotropic. At small departures from equilibrium the perturbation to $g(r)$ is linear in $P e$ and thus must be proportional to $P$ e $\hat{\boldsymbol{r}} \cdot\langle\hat{\boldsymbol{E}}\rangle \cdot \hat{\boldsymbol{r}}$. This perturbation will contribute to the shear viscosity but not to normal stress differences (Batchelor 1977). Only at the next order in $P e$ will normal stress differences be generated (Brady \& Vicic 1995). A positive $N_{1}$ arises from the negative $y y$ Brownian stress generated along the compressive axis of the flow. Brownian motion acts to repel two particles that are pushed together 
near contact by the shear flow, and the component of this repulsion along the $y$ axis is a positive $y y$-pressure or a negative stress. A similar Brownian component exists along the $x$-axis, and to $O(\mathrm{Pe})$ these two components are equal resulting in zero normal stress differences. As the Péclet number is increased the symmetry along the compressive axis is broken (cf. figure 8) resulting in a larger magnitude $y y$-stress and a positive $N_{1} . N_{2}$ is negative for the same reason - the $y y$-stress is larger in magnitude than the $z z$-stress because of the compression of the shear flow. The signs of the stresses are reversed along the extensional axis, but there are fewer near pairs as seen in figure 8 so the stress is dominated by the behaviour along the compressive axis. A similar explanation can be applied to polymeric systems.

This close analogy to polymeric systems can be further exploited to propose a stress-optical rule for colloidal dispersions. The birefringence of a colloidal suspension, like a polymer solution, is proportional to $\langle\hat{r} \hat{r}\rangle$; thus, the Brownian stress should be proportional to the birefringence. This analogy was first proposed by Wagner $\&$ Ackerson (1992) and used by Wagner to determine experimentally the separate hydrodynamic and Brownian contributions to the shear viscosity (Bender \& Wagner 1995).

As the Peclet number is increased the structural deformation increases and the Brownian contribution to the normal stresses increases. A maximum is reached for $P e \approx 1$ because the structural deformation cannot keep up with the flow, just as was the case for the shear viscosity. The Brownian contribution then ultimately decays to zero as the hydrodynamic limit is approached, again as $1 / P e$ according to the analysis of Morris \& Brady $(1996 b)$. In the range $1<P e<100$ the Brownian contribution to the normal stresses apparently fluctuates owing to the evolving ordered microstructure, although the scattered results for larger system sizes indicates that the statistics for $N_{1}$ and $N_{2}$ may not be very good in this region of $P e$.

The hydrodynamic contribution to the normal stresses is much smaller than the Brownian at low $P e$, and increases in magnitude as $P e$ increases. As a result, $N_{1}$ changes sign for $P e>O(10)$, a direct manifestation of the importance of hydrodynamic interactions. In the pure hydrodynamic limit $\left(P e^{-1} \equiv 0\right)$ one would expect the normal stresses to be zero from symmetry requirements. However, the singular boundary-layer behaviour as $P e \rightarrow \infty$ results in an asymmetric pair-distribution function at contact, which can be clearly seen in figure 10 for $P e=10^{3}$, and an $O(\eta \dot{\gamma})$ hydrodynamic normal stress difference. Again we see the important singular effect of Brownian motion at high Péclet number. Actually, the theory of Morris \& Brady (1996b) shows that for perfect hard spheres with hydrodynamics and Brownian motion only, the asymmetry vanishes as $P e^{-0.22}$ as $P e \rightarrow \infty$. The pure hydrodynamic limit of hard spheres is a special, singular case. If there are repulsive interparticle forces, no matter how small or short ranged, the asymmetry persists as $P e \rightarrow \infty$. Numerically, it is difficult to resolve the thin boundary layer at particle contact, and this inability may manifest itself as if an interparticle force were present. In an actual experimental situation no particles behave as true hard spheres, and we should expect finite normal stress differences.

Unfortunately there are no experimental data for normal stress differences for colloidal dispersions with which to compare our simulations.

Although the particle contribution to the stress defined in (2.8) is not traceless, the hydrodynamic functions necessary to compute the trace and therefore the evolution of the osmotic pressure with $\mathrm{Pe}$ were not available at the time these simulations were performed. The necessary functions were determined in Jeffrey, Morris \& Brady (1993) and will be used in future simulations. 


\subsection{Diffusion}

In table 2 we show the average translational and rotational mobilities of a particle, $D_{0}^{s}$ and $D_{0}^{r}$, respectively, as a function of $P e$. At $P e \equiv 0$ these instantaneous mobilities (multiplied by $k T$ ) correspond to the short-time self-diffusion coefficients. The mobilities have been normalized by the infinite-dilution self-diffusion coefficients $k T / 6 \pi \eta a$ and $k T / 8 \pi \eta a^{3}$, respectively. These values are averages of the separate $x-, y$ and $z$-components. The individual rotational mobilities are all identical to within the statistical uncertainty, while the $y y$ - and $z z$-components of the translation mobility are identical and the $x x$-component is perhaps $10 \%$ larger. The local mobility is to a very good approximation isotropic despite the structure formed during flow. The most important feature to note is that the mobilities remain roughly constant until the suspension starts to shear thicken, after which they decrease monotonically with increasing $P e$. This decrease is a manifestation of the closely touching particle clusters that form hindering the local motion of a particle.

The mean-square displacement of a Brownian particle is convectively enhanced by flow. For a simple shear flow the mean-square displacement is expected to grow in time according to (Elrick 1962)

$$
\begin{aligned}
\left\langle x^{2}(t)\right\rangle & =2 D_{x x} t+2 D_{y y} t\left[1+\frac{1}{3}(\text { Pe } t)^{2}\right], \\
\left\langle y^{2}(t)\right\rangle & =2 D_{y y} t \\
\left\langle z^{2}(t)\right\rangle & =2 D_{z z} t \\
\langle x(t) y(t)\rangle & =D_{y y} t(P e t),
\end{aligned}
$$

as $t \rightarrow \infty$. The vorticity $(z z)$ component is uncoupled from the flow and behaves normally, growing linearly in time. However, diffusion in the velocity-gradient direction $(y y)$ gives not only normal diffusive behaviour in the $y$-direction, but also couples to the motion in the $x$-direction, resulting in the $x$-displacement growing cubically in time with proportionality constant given by $D_{y y}$. Equations $(3.4 a-d)$ are used to determine the long-time self-diffusivities: the mean-square displacement from a simulation is plotted as a function of time, the proper growth law is substantiated to verify that the motion is indeed diffusive, and the coefficients $D_{y y}$ and $D_{z z}$ are extracted from the data. Because the $x$-displacement is dominated by the convective dispersion, growing as $t^{3}$, it is not possible to determine the $D_{x x}$ coefficient.

The Péclet-number dependence of $D_{y y}$ and $D_{z z}$ normalized by the diffusion coefficient of an isolated particle are shown in figure 7. As $P e \rightarrow 0$ the diffusivities approach the long-time self-diffusivities of equilibrium dispersions and agree with the experimental measurements of van Megen et al. (1986) and the theoretical prediction of Brady (1994). The action of flow is to enhance the self-diffusivity, with the leading correction scaling as $P e^{3 / 2}$ (Morris \& Brady 1996a). The increase in the self-diffusivities continues throughout the shear-thinning region of the viscosity curve (cf. figure 2). For $P e>3$ both diffusivities plunge, $D_{y y}$ dramatically, as the suspension orders. Indeed the sharp decrease in the diffusivities is a simple and direct indication of order formation.

As the Péclet number increases further, the disorder is disrupted and the selfdiffusivities grow in magnitude. The results show that the normalized self-diffusivities grow approximately linearly with $P e$ as $P e \rightarrow \infty$, or in dimensional terms $D_{y y} \sim \dot{\gamma} a^{2}$, corresponding to hydrodynamic diffusion. The simulation results for the shearinduced or hydrodynamic diffusivities are in reasonable agreement (within a factor of 2) with the experiments of Eckstein, Bailey \& Shapiro (1977), Leighton \& Acrivos 


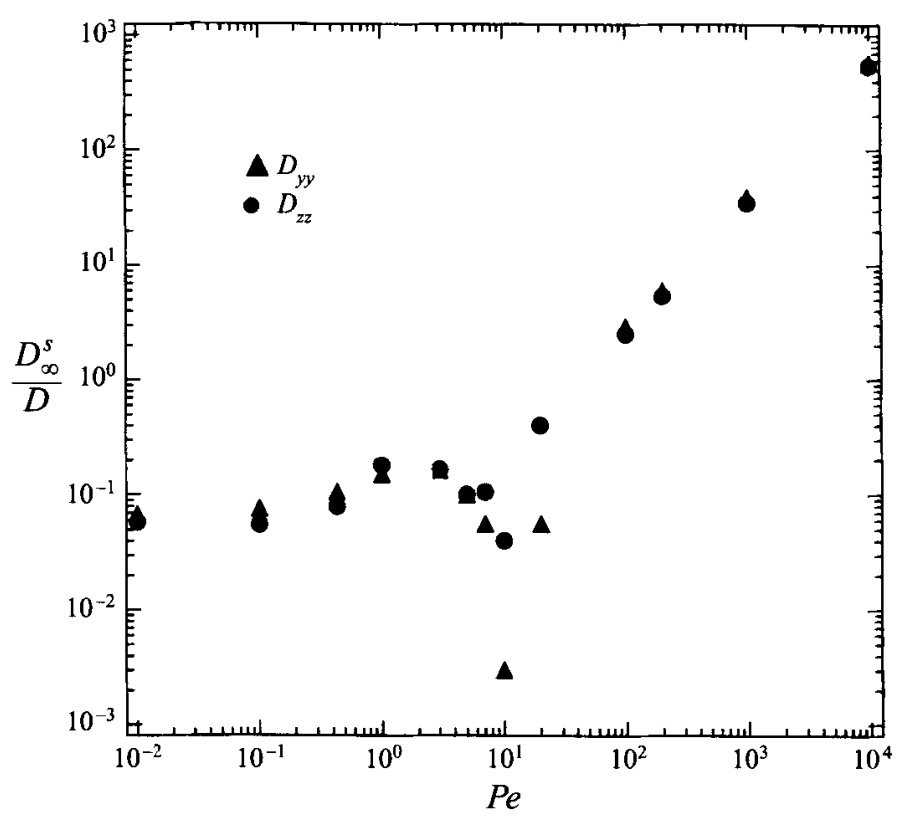

FIGURE 7. The long-time self-diffusivities in the velocity-gradient $D_{y y}$ and vorticity $D_{z z}$ directions as a function of $P e$ for $N=27$. The diffusivities have been normalized by the diffusion coefficient of an isolated Brownian particle, $k T / 6 \pi \eta a$. At large $P e$ the dimensional long-time diffusivities scale hydrodynamically as $\dot{\gamma} a^{2}$.

(1987) and Phan \& Leighton (1993). Again, the singular effect of Brownian motion is important in leading to diffusive behaviour as $P e \rightarrow \infty$. A pure hydrodynamic system is completely deterministic and, although the evolution equations for particle positions are highly nonlinear and give rise to deterministic chaos, the small amount of Brownian motion provides the necessary irreversibility for the chaos to set in. We were not able to determine long-time self-diffusivities from the simulations with $N>27$ as the runs were not long enough to reach the asymptotic temporal behaviour; thus, we do not know the effect of the size of the simulation cell on the diffusivities. The values obtained here are in agreement with the monolayer results of Bossis \& Brady (1987) when allowance is made for converting area fraction to volume fraction.

\subsection{Structure}

It is interesting to ask what is happening to the suspension structure throughout the shear thinning and thickening processes. There is a dramatic change in the structure as illustrated in figure 8 , where we show a projection of the pair-distribution function onto the plane of shear: light regions represent high probability and dark low. At small $P e$ we see clearly the distortion of the structure with an intensification and compression of the first nearest-neighbour peak at contact along the compressive axis of the flow and a corresponding diminution along the extensional axis. At intermediate Péclet numbers $(3<P e<200)$ we see the formation of an ordered structure with 'strings' of particles lined up along the flow direction. The projection of the pair-distribution function in the velocity-gradient-vorticity plane in figure 9 shows that the strings are arranged in an hexagonal pattern. After the first nearestneighbour peak along an individual string the particle positions are uncorrelated. This ordered structure does not appear to be an artifact of the simulation cell size, as 

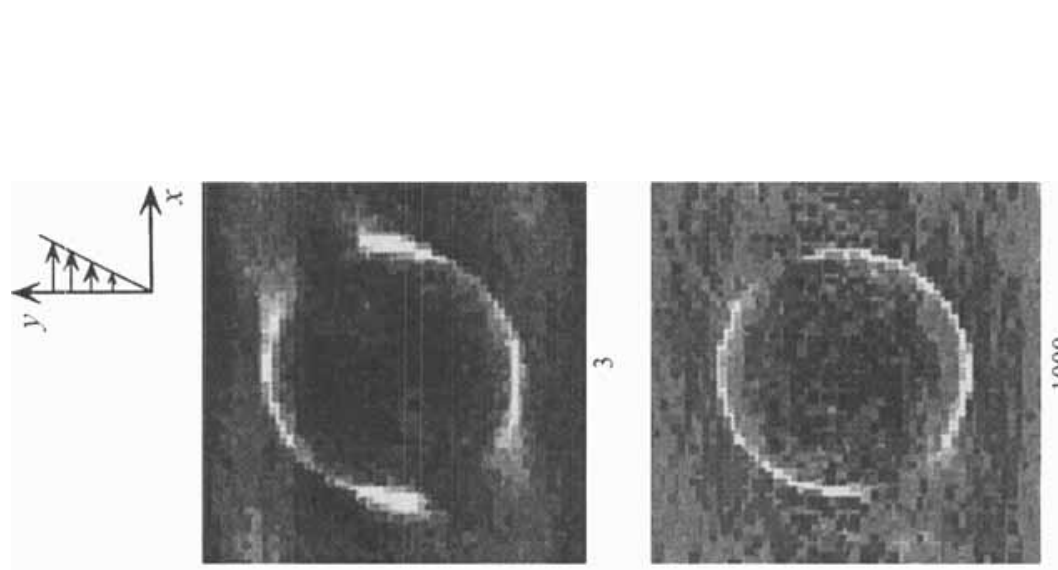

$\vec{g}$

至

春

㐘

은

영뭉

ב

퐆

8 :

힌응

2.

응

总

론

栆

तิ
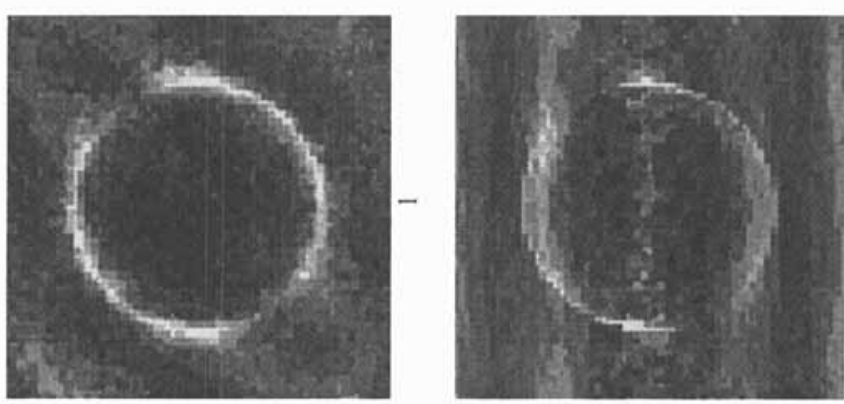

ㄱำ 옹

z

는

용

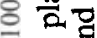

西

承

둥요

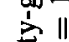

:

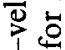
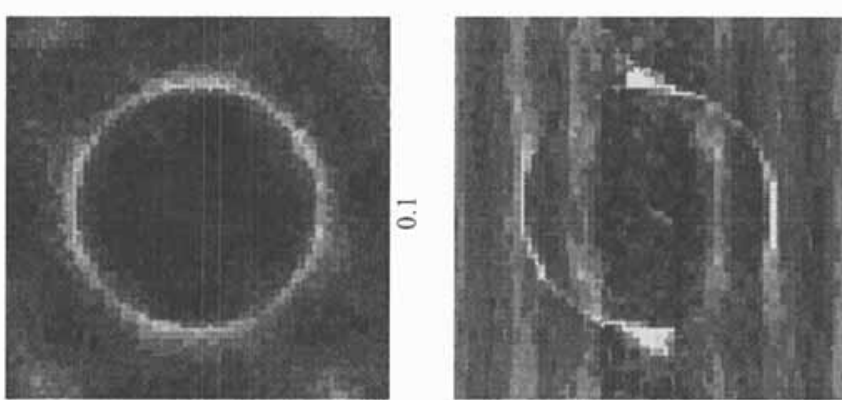

ㄷํㅇำ

$\stackrel{0}{\circ}$

:

릉

- 율

ㄱ. ำ

Z

.

중

혼

ล눙

क.

ำ
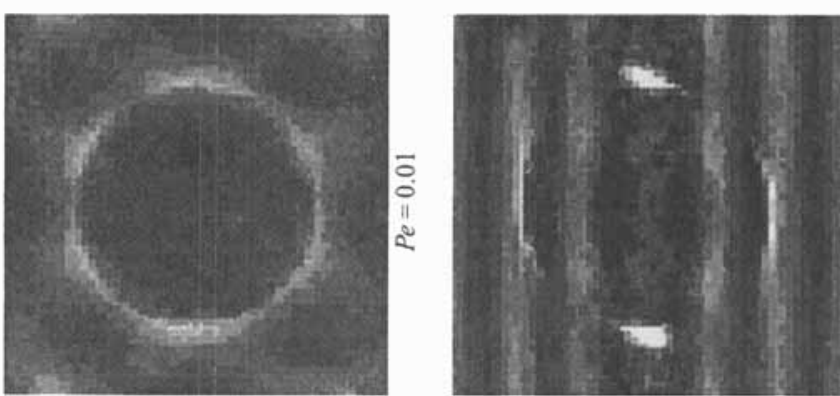

总

范

동

으 흘

"E

2 兽

壱

는

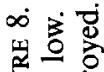

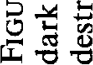



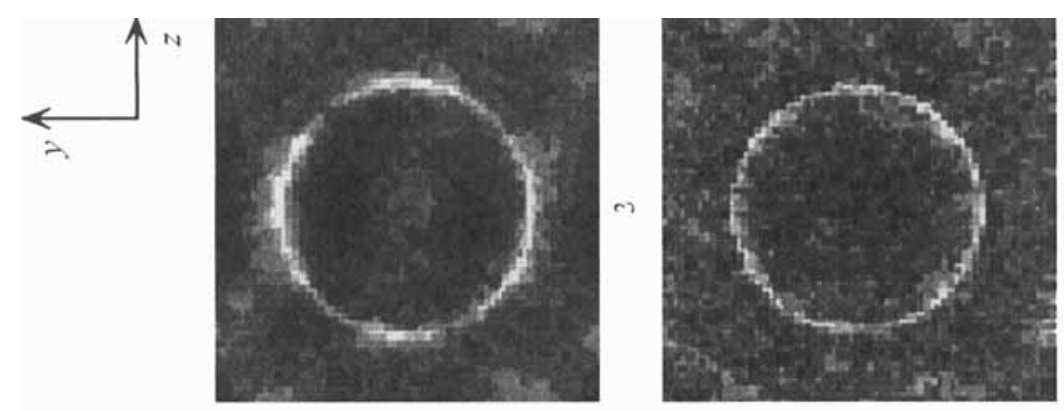

:

照

矛

웜

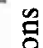

.



동음

동
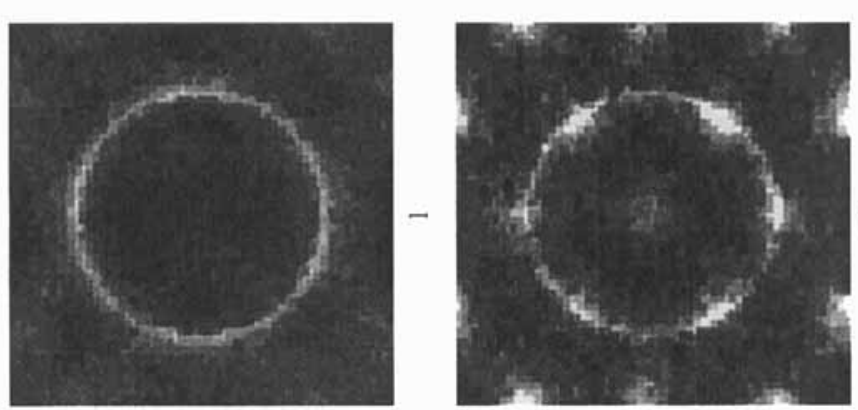

กิ

긍

$z$

눙

节

吾

咅

$8>0$

.들

낭

1

苞。

눌

so

$>0$
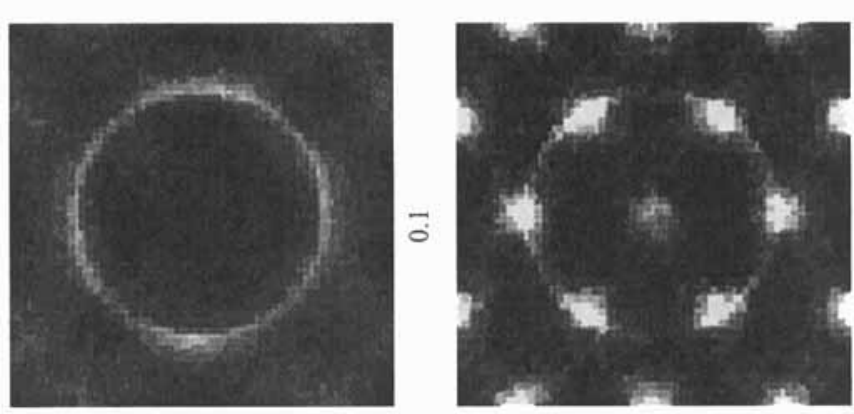

余

\%

폴

욤

유 듬 品 要

온융

I

is

.
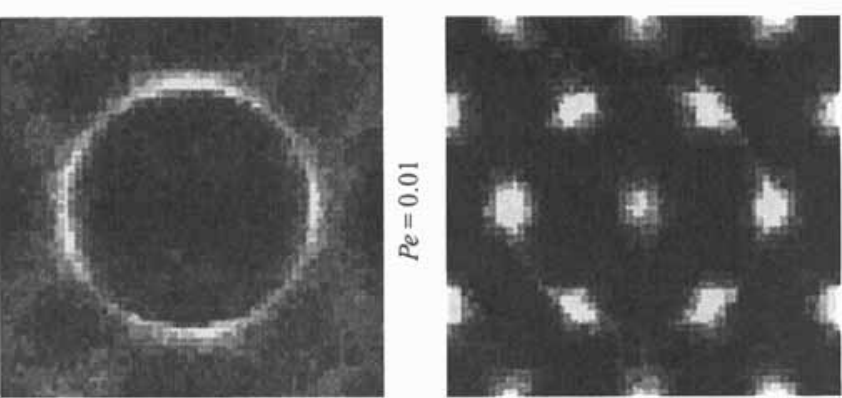

㞯

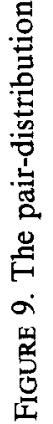




\section{High \\ Low}
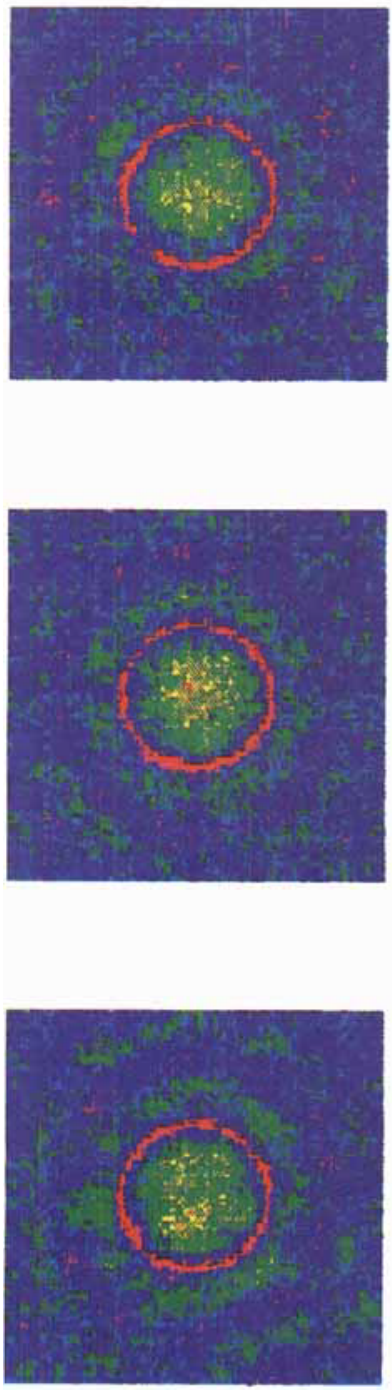

$P e=0.43$ $g(x, y)$

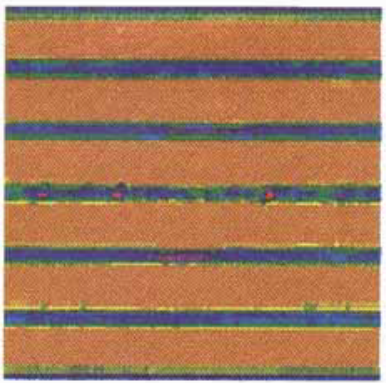

$g(x, z)$

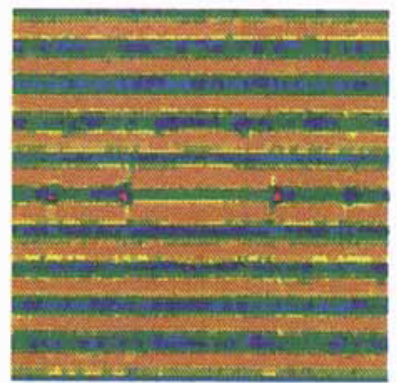

$g(z, y)$

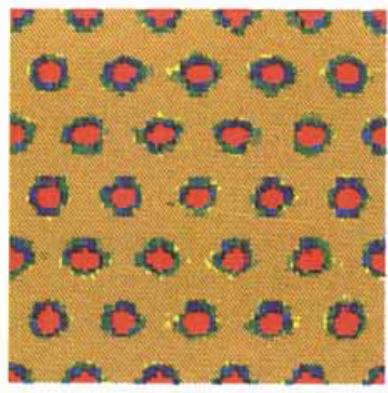

$P e=10$
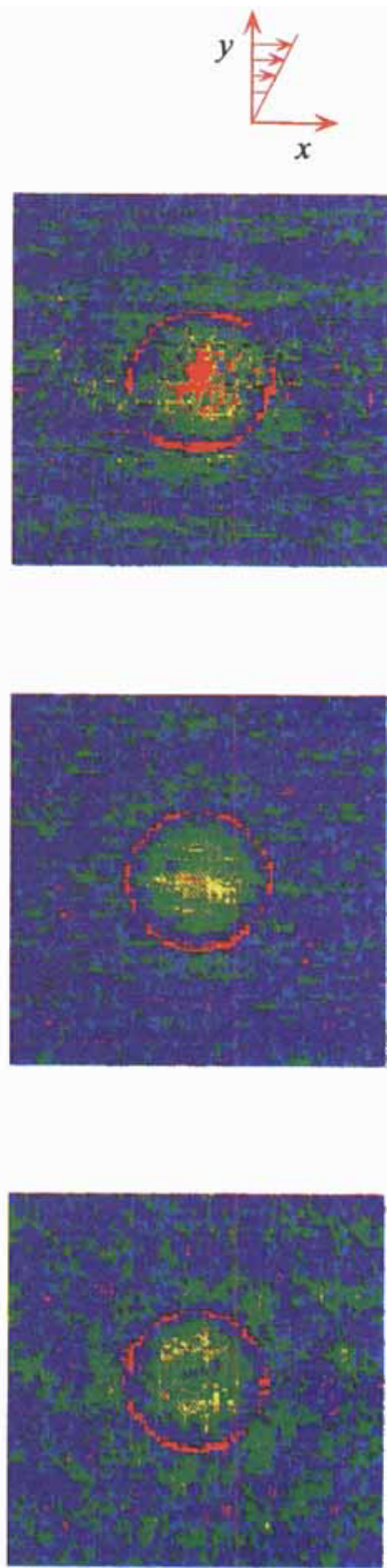

$$
P e=10^{3}
$$

FigURE 10. Projections of the pair-distribution function in all three planes for $N=123$ at $P e=0.43,10$, and 1000 .

simulations with 123 particles shown in figure 10 display the same pattern. Indeed, at $P e=10$ the ordering is very robust: simulations with $N=81$, which is not a number that can order easily into a cubic unit cell (unlike 27,64 or 123 , which correspond to $3 \times 3 \times 3,4 \times 4 \times 4$, and $5 \times 5 \times 5$, respectively), form a tilted hexagonal string pattern seen in figure 11 so as to accommodate the $4 \times 4 \times 5$ arrangement of particles.

The origin of the ordering can be explained as follows. At low $P e$ the randomizing effect of Brownian motion dominates and the suspension is a disordered liquid (cf. 


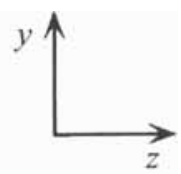

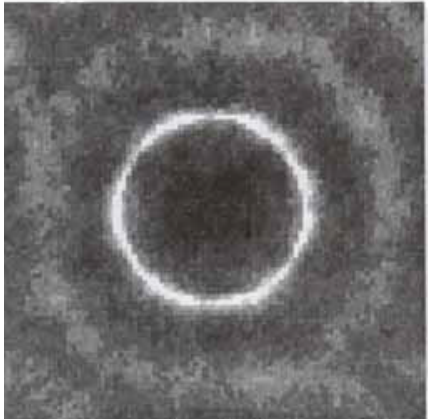

$P e=0.01$

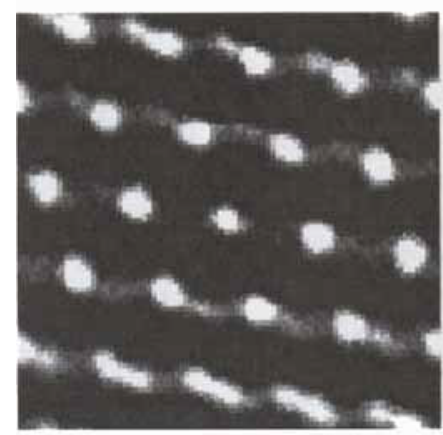

$P e=10$

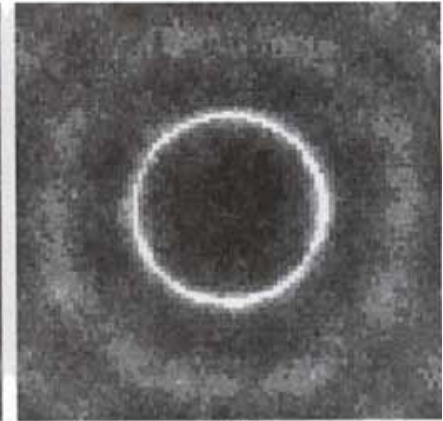

0.43

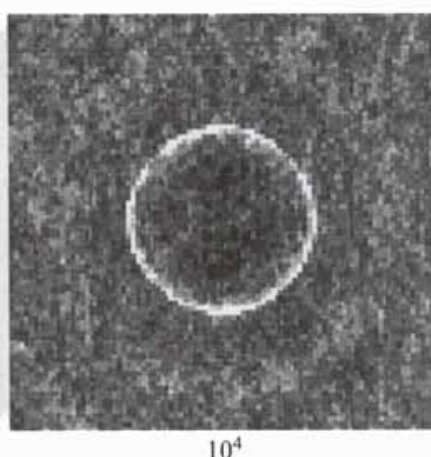

$10^{4}$

FIGURE 11. Projections of the pair-distribution function $g(z, y)$ in the velocity-gradient-vorticity plane for $N=81$ at $P e=0.01,0.43,10$, and $10^{4}$.

figure 11 at $P e=0.01$ ): flow is a weak perturbation to the equilibrium structure. For $P e \approx 10$ Brownian and hydrodynamic forces are comparable, and observations from animations of particle motion show that there is very little 'random' motion due to Brownian forces. The important effect of Brownian motion comes from the spatial variation of the relative mobility, $\nabla \cdot \boldsymbol{R}_{F U}^{-1}$, which acts as a short-range repulsive force between particles (Bossis \& Brady 1987). As two particles are convected towards each other in the flow, they 'collide', are pushed apart by the repulsive interaction, and recede from one another along trajectories that are displaced as illustrated in figure 12. Repeated collisions with subsequent particles leads to a separation of the particles into layers or strings along the flow direction.

This mechanism for ordering is not specific to Brownian suspensions: any system with simple shear flow competing against repulsive forces should give rise to a similar ordering. Indeed, we reported just such an ordering in our first paper on Stokesian Dynamics (Bossis \& Brady 1984) for a suspension of non-Brownian particles interacting through hydrodynamic and repulsive colloidal forces. That study was restricted to a monolayer and although strings did form in the flow direction we could not see the hexagonal pattern in the third direction. In the same year Erpenbeck (1984) reported string formation in a non-equilibrium molecular dynamics (NEMD) simulation of hard spheres in simple shear flow. Subsequently, many researches have 

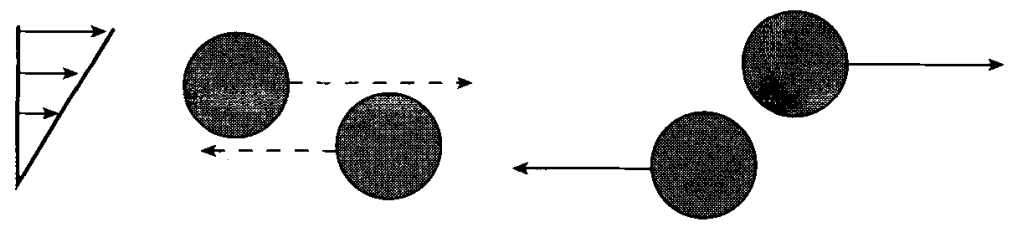

Figure 12. Collision of two particles in simple shear flow with repulsive interparticle forces (or the effect of Brownian forces through $\nabla \cdot \boldsymbol{R}_{F U}^{-1}$ ) showing the net displacement that occurs between the approaching and receding trajectories.

observed shear-induced string ordering in NEMD of hard and soft spheres (Hess 1985; Heyes 1986) and Brownian dynamics simulations with a Yukawa-type repulsive force (Heyes 1988; Xue \& Grest 1990). Brownian dynamics is Stokesian Dynamics without hydrodynamic interactions, specifically setting $\boldsymbol{R}_{F U}=\boldsymbol{I}$, and $\boldsymbol{R}_{F E}=\boldsymbol{R}_{S U}=\boldsymbol{R}_{S E}=0$ in (2.2)-(2.9).

This string-like order has also been observed experimentally. Ackerson (1990) carried out light scattering experiments on model hard-sphere colloidal suspensions for a range of volume fractions $(0.412<\phi<0.595)$ and Péclet numbers $\left(0.1<P e<10^{4}\right)$. At a volume fraction of 0.47 he observed an amorphous liquid-like state at low $P e$, a string-like order for $P e \approx 15$, and an amorphous liquid-like state for large $P e$, in complete agreement, even in the range of $P e$, with the results of this study. String-like order has also been observed by Laun et al. (1992) in scattering experiments under flow.

As the Péclet number is increased the ordered structure 'melts'. This melting appears to be gradual rather than sudden. It should be noted that all simulations were started at the equilibrium liquid-like configuration and the $P e$ suddenly increased to the final steady value. A few runs were made starting with the ordered state at a $P e$ between 10 and 100 and the shear rate increased to see if an already ordered suspension would maintain its order to a higher shear rate. In all cases the transition from order to disorder occurred at the same Péclet numbers. Experiments in which the shear rate was reduced from a high-shear-rate disordered state to a lower ordered shear rate followed the same behaviour as increasing the shear rate: the structure displayed no hysteresis. Also, as seen in the figures the order occurred throughout the simulation cell and was perfect. It is not known whether a very large simulation cell would simultaneously display regions of order and disorder, or have defects throughout the structure. It is also not known if a large system would display sharp disorder--order or order-disorder transitions.

The melting that occurs as the shear rate is increased is caused by the lubrication forces. For $\mathrm{Pe}>O(100)$ particles are brought close together forming a temporary doublet that rotates en mass and disrupts the string order. The ultimate state at high $P e$ is a disordered liquid with a sharpening of the first nearest-neighbour peak (compare figure 11 at $P e=0.01$ and $P e=10^{4}$ ) as hydrodynamic clusters form.

Thus we have an interesting flow-induced structural transition from disorder to order to disorder as the Péclet number is increased. At low volume fractions $(\phi<0.4)$ we did not observe any order formation: the suspension was always liquid-like (Phung et al. 1996a). At volume fractions above the equilibrium phase boundary $(\phi=$ $0.494)$ the suspension followed the sequence order-disorder-string-order-disorder as the Péclet number increased (Phung 1993; Phung et al. 1996b). Both of these observations agree with the experiments of Ackerson (1990). An interesting and unanswered question is whether this flow-induced disorder-order-disorder transition 


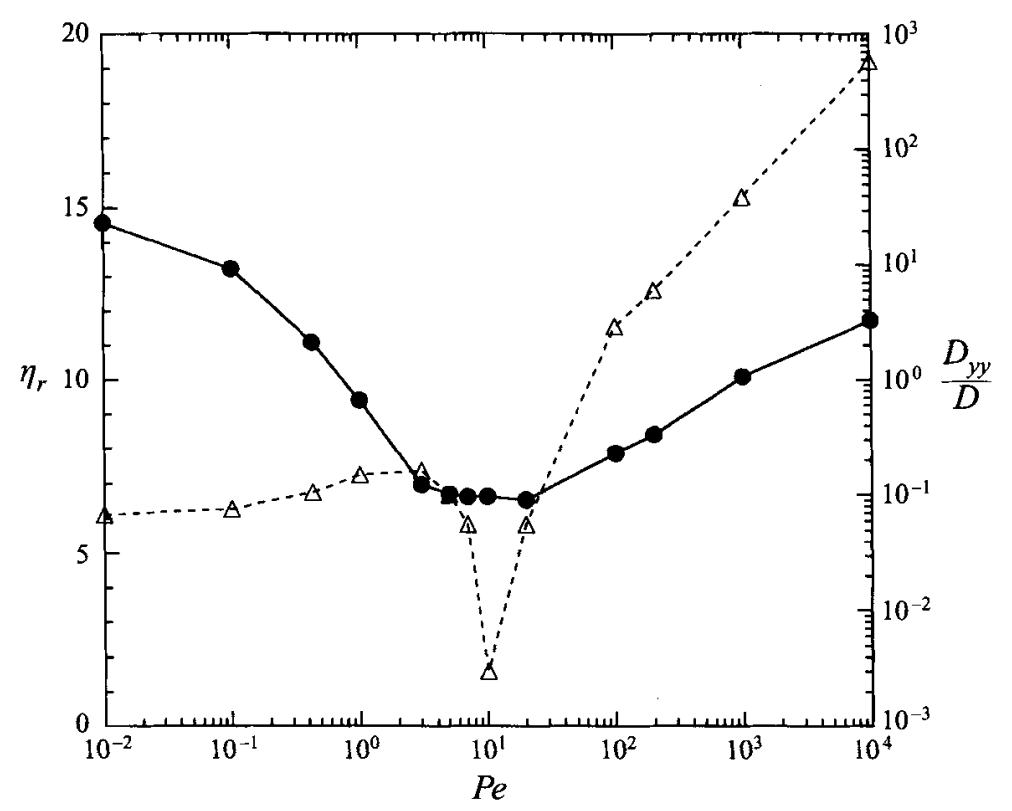

FIGURE 13. Comparison of the relative viscosity and the long-time self-diffusivity as a function of $P e$ for $N=27$. The shear thinning is not related to the ordered structure formed.

is a perturbation of the equilibrium phase boundary or represents a completely new non-equilibrium phase transition.

Two comments about the string ordering in relation to the suspension viscosity are in order. First, it is tempting to associate the flow-induced ordering with shear thinning. Arguments have been advanced to suggest that the suspension orders so that it can flow more easily, leading to a reduction in the suspension viscosity. In figure 13 we simultaneously show the suspension viscosity and long-time self-diffusivity as a function of $P e$. One sees quite clearly that throughout the shear thinning process the diffusivity actually increases - the suspension is far from being ordered. The ordering occurs after the shear thinning is over. Suspensions at lower volume fraction also shear thin but do not order. There is no necessary relationship between shear thinning and ordering.

Second, there is also no necessary relationship between the disruption of the ordered structure and shear thickening. It is true that the ordered structure must be destroyed for hydrodynamic clusters to form and the viscosity to increase. However, the viscosity in the ordered state in figure $\mathbf{1 3}$ is the same as in a disordered fluid at the same $\mathrm{Pe}$. Simply going from an ordered to a disorder state will not necessarily increase the viscosity. Simulations at lower volume fractions show no order, but still shear thicken. What is necessary is to go from a 'well dispersed' state, for which an ordered state qualifies, to a state with non-compact hydrodynamic clusters. Further, one should note that the increase in viscosity comes from the hydrodynamic component only, and the only way to change this is to form non-compact clusters as demonstrated earlier. This does not mean that one cannot get shear thickening from the disruption of an ordered structure, only that it is not necessary to have an ordered state to give rise to shear thickening. 


\section{Conclusions}

In this paper we have investigated the non-equilibrium behaviour of concentrated colloidal dispersions of hard spheres in simple shear flow by Stokesian Dynamics simulation. The suspension is governed by the competition between Brownian and hydrodynamic forces as measured by the Péclet number. At low Péclet number the perturbation to the equilibrium structure is small, but the suspension shear thins dramatically. This shear thinning results from the decrease of the direct Brownian contribution to the stress as the structural deformation cannot keep up with the shear flow. The hydrodynamic contribution to the viscosity remains constant and equal to the high-frequency dynamic viscosity throughout the shear thinning process.

At higher Péclet numbers hydrodynamic and Brownian forces balance to produce a string-ordered structure. In the ordered state the stresses remain constant, but the long-time self-diffusivity drops dramatically as the particle motion is constrained to lie along the strings. At still higher shear rates $(P e>200)$ the strings melt and the suspension becomes a disordered liquid. Owing to the strong hydrodynamic lubrication forces large non-compact clusters form whose characteristic size grows as the Péclet number is increased. As the cluster size grows the viscosity increases and the suspension shear thickens. This shear thickening is hydrodynamic in origin: hydrodynamics cause the clusters to form and the stress is purely hydrodynamic at large Péclet numbers.

In the infinite-Péclet-number limit a suspension of perfect hard spheres appears to be singular in that a small amount of Brownian motion or interparticle forces has a dramatic effect on structure and properties. As shown by Morris \& Brady (1996b) there is a thin $O\left(P e^{-1}\right)$ boundary layer at particle contact in which hydrodynamic, interparticle and Brownian forces balance and lead to an asymmetry in the pairdistribution function and a loss of flow reversal symmetry. This asymmetry produces $O(\eta \dot{\gamma})$ hydrodynamic normal stress differences and $O\left(\dot{\gamma} a^{2}\right)$ shear-induced particle diffusivities in the infinite- $P e$ limit. This residual affect of Brownian motion may also contribute to setting the ultimate size of the hydrodynamic clusters - and hence the suspension viscosity - that form at high shear rates.

Although we have only studied the simplest suspension - hard spheres with hydrodynamics and Brownian motion, the behaviour we have observed is generic to systems with repulsive interparticle forces. A repulsive interparticle forces acts in much the same way as the spatial variation in the relative mobility $\nabla \cdot \boldsymbol{R}_{F U}^{-1}$. Completely analogous behaviour in terms of shear thinning and thickening and ordered structure formation has been seen in colloidal dispersions with repulsive forces. Indeed, one can often simply scale the behaviour of repulsive systems onto that of hard spheres by using an equivalent volume fraction that incorporates the short-range repulsion into an effective radius of a particle (Ackerson 1990; Brady 1993b). Long-range repulsive forces are somewhat different in that hydrodynamic interactions are minimized or absent, which results in a different scaling for the dependence of the viscosity on concentration (Brady 1993b), but the shear thinning and ordering phenomena are still present.

Although the results we have presented here are for small system sizes they appear to agree quantitatively with experiment for viscosity and structure formation. There are no measurements of normal stresses or diffusivities, however. These results give confidence that Stokesian Dynamics can be used to quantitatively study the behaviour of colloidal dispersions over a wide range of conditions. In a companion set of papers 
(Phung et al. 1996a,b) we report on simulation results for lower and higher volume fractions of Brownian suspensions of hard spheres.

The work was supported in part by grants CTS-9020646, CTS-9420415, and INT9415673 from the National Science Foundation and by an allocation of computer time at the San Diego Supercomputer Center.

\section{REFERENCES}

ACKERSON, B. J. 1990 Shear induced order and shear processing of model hard sphere suspensions J. Rheol. 34, 553.

Ackerson, B. J. \& Pusey, P. N. 1988 Shear-induced order in suspensions of hard spheres. Phys. Rev. Lett. 61, 1033.

BATCHelor, G. K. 1977 The effect of Brownian motion on the bulk stress in a suspension of spherical particles. J. Fluid Mech. 83, 97.

BENDER, J. W. \& WAGNer, N. J. 1995 Optical measurement of the contribution of colloidal forces to the rheology of concentrated suspensions J. Colloid Interface Sci. 172, 171.

Bossis, G. \& BRady, J. F. 1984 Dynamic simulation of sheared suspensions. I. General method. $J$. Chem. Phys. 80, 5141.

Bossis, G. \& BRADY, J. F. 1987 Self-diffusion of Brownian particles in concentrated suspensions under shear. J. Chem. Phys. 87, 5437.

Bossis, G. \& Brady, J. F. 1989 The rheology of Brownian suspensions. J. Chem. Phys. 91, 1866.

Bossis, G., Meunier, A. \& Brady, J. F. 1991 Hydrodynamic stress of fractal aggregates of spheres. J. Chem. Phys. 94, 5064.

Brady, J. F. 1993 a Brownian motion, hydrodynamics, and the osmotic pressure. J. Chem. Phys. 98, 3335 .

BRadY, J. F. $1993 b$ The rheological behaviour of concentrated colloidal dispersions. J. Chem. Phys. 99, 567.

BRADY, J. F. 1994 The long-time self-diffusivity in concentrated colloidal dispersions. J. Fluid Mech. 272, 109.

Brady, J. F. \& Bossis, G. 1985 The rheology of concentrated suspensions of spheres in simple shear flow by numerical simulation. $J$. Fluid Mech. 155, 105.

Brady, J. F. \& Bos is, G. 1988 Stokesian dynamics. Ann. Rev. Fluid Mech. 20, 111.

Brady, J. F., Lester, J., Phillips, R. \& Bossis, G. 1988 Dynamic simulation of hydrodynamically interacting suspensions. $J$. Fluid Mech. 195, 257.

Brady, J. F. \& VICIC, M. 1995 Normal stresses in colloidal dispersions. J. Rheol. 39, 545.

Dhaene, P., Mewis, J. \& Fuller, G. G. 1993 Scattering dichroism measurements of flow-induced structure of a shear thickening suspension. J.Colloid Interface Sci. 156, 350.

Durlofsky, L. J., Brady, J. F. \& Bossis, G. 1987 Dynamic simulation of hydrodynamically interacting particles. J. Fluid Mech. 180, 21.

Eckstein, E. C., Bailey, D. G. \& Shapiro, A. H. 1977 Self-diffusion in shear flow of a suspension. J. Fluid Mech. 79, 191.

ELRICK, D. E. 1962 Source functions for diffusion in uniform shear flow. Austral. J. Phys. 15, 283.

ERPENBECK, J. J. 1984 Shear viscosity of the hard-sphere fluid via nonequilibrium molecular dynamics. Phys. Rev. Lett. 52, 1333.

Grassia, P. S., Hinch, E. J. \& Nitsche, L. C. 1995 Computer simulations of Brownian motion in complex systems. J. Fluid Mech. 282, 373.

Hess, S. 1985 Shear-induced melting and reentrant positional ordering in a system of spherical particles. Intl J. Thermophys. 6, 657.

Heyes, D. M. 1986 Some physical consequences of large shear rates on simple fluids. J. Chem. Phys. 85, 997.

Heyes, D. M. 1988 Shear thinning of dense suspensions modeled by Brownian dynamics. Phys. Lett. 132, 399.

JefFrey, D. J., Morris, J. F. \& Brady, J. F. 1993 The pressure moments for two rigid spheres in low-Reynolds-number flow. Phys. Fluids A 5, 2317. 
Kruif, C. G. De, Iersel, E. M. F. van, VRIJ, A. \& Russel, W. B. 1985 Hard sphere colloidal dispersion: viscosity as a function of shear rate and volume fraction. J. Chem. Phys. 83, 4717 .

Laun, H. M., Bung, R., Hess, S., Loose, W., Hess, O., Hahn, K., Hädicke, E., Hingmann, R., SCHMIDT, F. \& LiNDER, P. 1992 Rheological and small angle neutron scattering investigation of shear-induced particle structures of concentrated polymer dispersions submitted to plane Poiseuille and Couette flow. J. Rheol. 36, 743.

LEIGHTON, D. \& ACRIVOS, A. 1987 Measurement of self-diffusion in concentrated suspensions of spheres. J. Fluid Mech. 177, 109.

MACKAY, M. E. \& KAFFASHI, B. 1995 Stress jumps of colloidal suspensions, measurements of the elastic-like and viscous-like stress components. J. Colloid Interface Sci. 174, 117.

Megen, W. van, UnderwoOd, S. M. \& SNOOK, I. 1986 Tracer diffusion in concentrated colloidal dispersions. J. Chem. Phys. 85, 4065.

Morris, J. F. \& Brady, J. F. $1996 a$ Self-diffusion in sheared suspensions. J. Fluid Mech. 312, 223-252.

Morris, J. F. \& Brady, J. F. $1996 b$ Microstructure of strongly-sheared suspensions and its impact on rheology and diffusion. J. Fluid Mech. (to be submitted).

O'BRIEN, R. W. 1979 A method for the calculation of the effective transport properties of interacting particles. J. Fluid Mech. 56, 401.

Phan, S. E. \& Leighton, D. L. 1993 Measurement of the shear-induced tracer diffusivity in concentrated suspensions. J. Fluid Mech. (submitted).

Phillips, R., Brady, J. F. \& Bossis, G. 1988 Hydrodynamic transport properties of hard-sphere dispersions. I. Suspensions of freely mobile particles. Phys. Fluids 31, 3462.

Phung, T. N. 1993 Behaviour of concentrated colloidal suspensions by Stokesian Dynamics simulation. PhD Thesis, California Institute of Technology.

Phung, T. N. \& BradY, J. F. 1992 Microstructured fluids: structure, diffusion and rheology of colloidal dispersions. In Slow Dynamics in Condensed Matter (ed. Kawasaki, Tokuyama \& Kawakatsu), p. 391. AIP Conference Proc. 256.

Phung, T. N., Brady, J. F. \& Bossis, G. 1996a Stokesian Dynamics simulation of colloidal dispersions. I. Moderately concentrated hard-sphere suspensions. J. Rheol. (to be submitted).

Phung, T. N., Brady, J. F. \& Bossis, G. $1996 b$ Stokesian Dynamics simulation of colloidal dispersions. II. Very concentrated hard-sphere suspensions. J. Rheol. (to be submitted).

Pusey, P. N. 1991 Colloidal suspensions. In Liquids, Freezing and Glass Transition (ed. J. P. Hansen, D. Levesque \& J. Zinn-Justin). Elsevier.

Rallison, J. M. \& HiNCH, E. J. 1986 The effect of particle interactions on dynamic light scattering from a dilute solution. J. Fluid Mech. 167, 131 .

Thomas, D. G. 1965 Transport characteristics of suspensions. VIII. A note on the viscosity of Newtonian suspensions of uniform spherical particles. J. Colloid Interface Sci. 20, 267.

WAGNer, N. J. \& ACKERSON, B. J. 1992 Analysis of nonequilibrium structures of shearing colloidal suspensions. J. Chem. Phys. 97, 1473.

WERFF, J. C. VAN DER \& KRUIF, C. G. DE 1989 Hard-sphere colloidal dispersions: the scaling of rheological properties with particle size, volume fraction, and shear rate. $J$. Rheol. 33, 421.

Werff, J. C. van DeR, Kruif, C. G. DE, Blom, C. \& Mellema, J. 1989 Linear viscoelastic behaviour of dense hard-sphere dispersions. Phys. Rev. A 39, 67.

XuE, W. \& Grest, G. S. 1990 Shear-induced alignment of colloidal particles in the presence of a shear flow. Phys. Rev. Lett. 64, 419. 